\title{
Low-Frequency Broadband Sound Transmission Loss of Infinite Orthogonally Rib-Stiffened Sandwich Structure with Periodic Subwavelength Arrays of Shunted Piezoelectric Patches
}

\author{
Zhifu Zhang, ${ }^{1}$ Weiguang Zheng, ${ }^{2}$ and Qibai Huang ${ }^{1}$ \\ ${ }^{1}$ State Key Laboratory of Digital Manufacturing Equipment and Technology, School of Mechanical Science and Engineering, Huazhong \\ University of Science and Technology, Wuhan 430074, China \\ ${ }^{2}$ Electromechanical Engineering College, Guilin University of Electronic Technology, Guilin 541004, China
}

Correspondence should be addressed to Qibai Huang; qbhuang@hust.edu.cn

Received 16 December 2016; Revised 10 February 2017; Accepted 16 February 2017; Published 9 April 2017

Academic Editor: Lorenzo Dozio

Copyright (C) 2017 Zhifu Zhang et al. This is an open access article distributed under the Creative Commons Attribution License, which permits unrestricted use, distribution, and reproduction in any medium, provided the original work is properly cited.

\begin{abstract}
This paper studies low-frequency sound transmission loss (STL) of an infinite orthogonally rib-stiffened sandwich structure flexibly connected with periodic subwavelength arrays of finite shunted piezoelectric patches. A complete theoretical model is proposed by three steps. First, the panels and piezoelectric patches on both sides are equivalent to two homogeneous facesheets by effective medium method. Second, we take into account all inertia terms of the rib-stiffeners to establish the governing equations by space harmonic method, separating the amplitude coefficients of the equivalent facesheets through virtual work principle. Third, the expression of STL is reduced. Based on the two prerequisites of subwavelength assumption and convergence criterion, the accuracy and validity of the model are verified by finite element simulations, cited experiments, and theoretical values. In the end, parameters affecting the STL performance of the structure are studied. All of these results show that the sandwich structure can improve the low-frequency STL effectively and broaden the sound insulation bandwidth.
\end{abstract}

\section{Introduction}

Classical sandwich structure consists of two parallel panels (as the facesheets) reinforced by sets of periodic ribstiffeners (as the sound bridges) and filled with air, which can be found in a wide range of engineering applications, such as lightweight aircraft sidewall panels, submarine/ship constructions, and express trains [1-11]. In the low-frequency range, the classical sandwich structure can be modeled as an orthotropic plate when the rib-stiffener spacing is much smaller than a wavelength of the panel flexural wave. However, in the relatively high frequency range, the exact model of the rib-stiffeners should be taken into account in the case where the wavelength is comparable with rib-stiffener spacing $[2,6,10,11]$. In view of the vibroacoustic characteristics of sandwich structure, a large number of valuable literatures cover the corresponding theoretical models, simulations, or experiments.

For the study of sandwich structure, more than 20 theoretical models have been presented. More intuitively, only by the detailed comparison of Hongisto [12] can it be found that the prevalent models for the prediction of STL are as high as 16. According to the classical sandwich structure, the sound bridges will significantly reduce the sound insulation in the middle and high frequency bands, and three methods are commonly used in predicting the vibroacoustic response. The first method is the decoupled approach, which divides the sound transmission paths into a fluid-borne through the air cavity and a structure-borne through the bridges. The sound transmission coefficient of the fluid-borne path can usually be analyzed by the transfer matrix method (TMM) $[8,13]$; the pressures and velocities at the top and bottom 
facesheets of the sandwich structure form a four-terminal network. To solve the sound transmission coefficient of the structure-borne path, Sharp [14] assumed that the velocity of the first facesheet was unaffected by the connections, and the bridges were rigid and massless; then a theoretical model was proposed. But the shortcoming is that the STL curve was required to add a positive empirical correction of $5 \mathrm{~dB}$. Gu and Wang [15] extended Sharp's theory by modeling the bridge connections as equivalent translational springs, and then the sandwich structure was simplified as a classical mass-spring-mass system. The model developed by Fahy and Gardonio [16] has similarities and differences with Sharp's model, it is based on the assumption that the dynamic properties of each stud are independent of each other, and their motions are restricted to translation, caused by incident bending waves normal to the line connections between the facesheets and studs. Davy [17] relied on Fahy's theory; he supposed that the bridges have flexible properties and facesheets have different line impedances. Legault and Atalla [8] summed up the previous four theoretical models and obtained the conclusion that Davy's model is good over the whole frequency range $100 \mathrm{~Hz}-10 \mathrm{kHz}$ by comparing with the experimental values.

The second method is the Fourier transform technique, which has a wide range of applications. For example, Rumerman [18] proposed a general solution for the forced vibration problem of an infinite thin plate with periodically uniform rib-stiffeners, but this method only considers the tension forces and bending moments between the facesheet and ribstiffeners, and the impact of the torsional moments is ignored. Lin and Garrelick [19] calculated the sound transmission of a sandwich structure with rigid bridges. Takahashi [20] discussed the sound radiation of the stiffened sandwich structure with multipoint connections excited by mechanical point forces, but the governing equations did not consider the fluid loads. Recently, Maxit [21] studied the vibration and sound radiation of a periodically ribbed plate to a mechanical point force drive by an efficient method based on the Fourier transform technique. Xin and Lu [11] proposed a theoretical model to calculate the sound radiation from an infinite sandwich structure reinforced by orthogonal rib-stiffeners; it is worth mentioning that the motions of equally spaced rib-stiffeners are determined by considering their tensional forces, bending moments, and torsional moments.

The third method is a hybrid of space harmonic method and virtual work principle. In advance, Mead [22, 23] analyzed the responses of the structure by the space harmonic method and then studied the dynamics of a periodic unit of the system based on the virtual work principle. Further, Lee and Kim [6] proposed a theoretical analysis model based on the hybrid method, to calculate STL performances of a thin plate stiffened by equally spaced line stiffeners; each stiffener was simplified as a combination of a lumped mass and rotational and translational springs. Extending this model to sandwich structure stiffened with parallelly placed studs, Wang et al. [7] developed a periodic model and took account of the discrete placing of the studs. However, this model only included the effect of tensional forces and bending moments between the studs and facesheets and ignored the torsional moments. On the basis of the above two models, Xin and Lu [10] perfected a deterministic theoretical model by coupling the acoustic and structure vibration and studied the STL of an infinite sandwich structure reinforced by orthogonal ribstiffeners. All possible motions of the rib-stiffeners, such as tensional forces, bending moments, and torsional moments as well as the corresponding inertial terms, were taken into account in the governing equations of the two facesheets.

When the experiment of STL through sandwich structure was mentioned, the experimental results measured by the National Research Council of Canada (NRCC) were more comprehensive and authoritative, its internal report IRC-IR761 [24] contained 350 sets of measurements, and the experimental specimens were mostly single and double-ply gypsum board attached parallel to steel/wood/nonloadbearing steel studs. In the existing literatures on the STL of stiffened plate structure, the experimental results in some literatures $[25,26]$ were directly referenced to the measurement data in the internal report IRC-IR-761 of NRCC [24] and in some documents [8] are measured by the relevant research institutions. In addition, there are parts of the literatures based on experimental study; for example, a series of articles by Hongisto [27-30] measured the structural transmission through the door leaf, the leak transmission through the slits, and flanking transmission via double structures by sound intensity method or sound pressure method. Among them, the test laboratory, measurement equipment, test specimens, and test methods were described in detail.

Although there are many studies on the STL of sandwich structure, only the middle and high frequency bands have a good sound insulation effect, while the STL performance in low-frequency range is still not improved. Therefore, this work first introduces periodic subwavelength arrays of finite shunted piezoelectric patches into an infinite orthogonally rib-stiffened sandwich structure and aims to enhance the STL in the low-frequency range. However, the existing research on periodic subwavelength arrays of shunted piezoelectric patches is basically focused on the vibration control, as well as noise radiation from metamaterial-based thin plates [3135]; study into STL remains to be accomplished, not to mention the low-frequency broadband STL. For example, Xiao et al. [36] were concerned with STL of metamaterialbased thin plates consisting of multiple subwavelength arrays of spring-mass resonators and completed the prediction by two analytical wave approaches: the plane wave expansion (PWE) and effective medium (EM) method. Zhang et al. [37] introduced the piezoelectric shunt damping into the periodic subwavelength structure and developed EM method for calculation of STL, but it was insufficient that the connections between the piezoelectric patches and substrate are rigid.

In view of the infinite orthogonally rib-stiffened sandwich structure flexibly connected with periodic subwavelength arrays of finite shunted piezoelectric patches (hereafter termed research structure), the studies on STL prediction of the research structure are blank. Therefore, this work focuses on the low-frequency broadband STL prediction of the research structure and carries out studies from the following four aspects. First, a complete theoretical model of STL for solving the studied sandwich structure can be built based on 


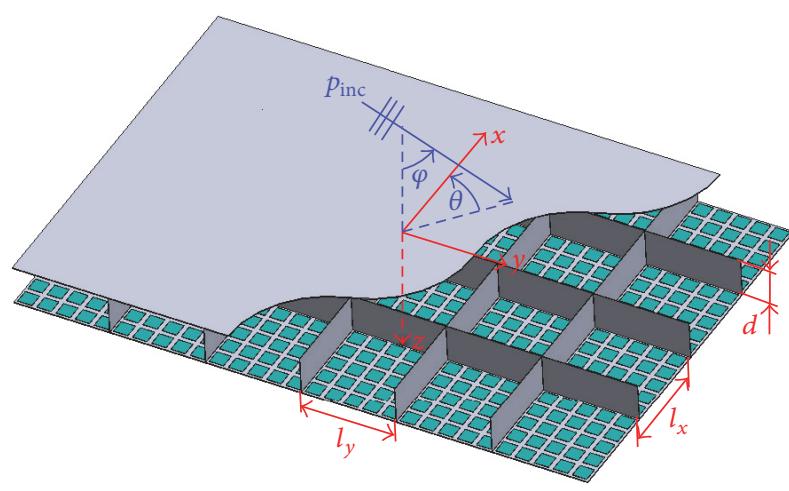

FIGURE 1: Research model of an orthogonally rib-stiffened sandwich structure with periodic subwavelength arrays of shunted piezoelectric patches.

the EM method, space harmonic method, and virtual work principle. Second, two prerequisites for the application of the theoretical model are given. Next, the accuracy of the theoretical model is verified by finite element simulation and cited experimental data, and the validity is verified by the comparison of the STLs before and after adding the shunt piezoelectric patches. Afterwards, the parameters affecting the STL performance of the research structure are studied.

\section{Theoretical Modeling of Vibroacoustic Response}

2.1. Preliminary Considerations. Considering the research model is an infinitely lager two-dimensional (2D) sandwich structure as shown in Figure 1. It consists of two infinite thin plates connected with periodic subwavelength arrays of shunted piezoelectric patches on the inner side and the two parallel facesheets reinforced by an orthogonally ribstiffened core which has two periodic uniform spacing of $l_{x}$ and $l_{y}$ in the $x$ - and $y$-directions, respectively. Its geometrical parameters are thickness of top and bottom plates $h_{1}$ and $h_{2}$, depth of orthogonal rib-stiffeners $d$, and thickness of $x$ and $y$-wise stiffeners $t_{x}$ and $t_{y}$, respectively. A right-handed Cartesian coordinate system $(x, y, z)$ is established on the upper surface of top plate, the positive direction of $z$-axis is vertical downward, and the positive directions of the $x$ and $y$-axis, are, respectively, along the two orientations of the orthogonal rib-stiffeners. The research structure divides the spatial sound field into three parts: the top sound field (semiinfinite sound field) in the area of $z<0$, the middle sound field (divided into periodic cavities by the orthogonal ribstiffeners) in between the area of $h_{1}<z<h_{1}+d$, and the bottom sound field (semi-infinite sound field) in the area of $z>h_{1}+d+h_{2}$.

An acoustic plane wave $P_{\text {inc }}$ varying harmonically in time impinging on the upper surface of the top plate is included in the sandwich structure. The incident plane wave induces motion of the top plate, and the motion creates a pressure inside the middle sound field and drives the deformations of the orthogonal rib-stiffeners. Therefore, the acoustic energy can travel from the top plate to the bottom plate via two separate paths: the fluid-borne path through the middle sound field and the structure-borne through the orthogonal rib-stiffeners. The incident wave makes an elevation angle $\varphi$ with the $z$-axis and its projection in the $x y$-plane makes an azimuth angle $\theta$ with the $x$-axis. Its amplitude is $P_{0}$ and the sound wavenumber vector $k_{0}$ can be decomposed in the $x$-, $y$-, and $z$-directions [8]:

$$
P_{\text {inc }}(x, y, z, t)=P_{0} e^{-i\left(k_{x} x+k_{y} y+k_{z} z-\omega t\right)},
$$

where

$$
\begin{aligned}
& k_{x}=k_{0} \sin \varphi \cos \theta, \\
& k_{y}=k_{0} \sin \varphi \sin \theta, \\
& k_{z}=k_{0} \cos \varphi,
\end{aligned}
$$

$$
k_{0}=\frac{\omega}{c_{0}} .
$$

$c_{0}$ in (2) is the sound speed in air.

2.2. Equivalent Characteristics of Periodic Facesheets. In view of the spatial periodicity of the research structure as shown in Figure 1, it is necessary to consider only the vibroacoustic response contribution from one period of element which is shown in the red cube box in Figure 2(a). Among them, assuming the rib-stiffener spacing $l_{x}=l_{y}$, that is, the top and bottom facesheets of one period are square structure, their inner surfaces each contain $a^{2}$ pieces of piezoelectric patches, with the formation of a periodic subwavelength structure of $a$ rows and $a$ columns (see Figure 2(b)). The yellow box is the basic unit of periodic subwavelength structure in Figure 2(a); its top view is shown in Figure 3(a), which is divided into a multilayer region $(\mathrm{m})$ and a single layer region (s); the lattice constant and size of piezoelectric patch are, respectively, $l$ and $l_{p}$.

The flexible connections between the piezoelectric patches and facesheets can be initially equivalent to a spring and a damper in a unit cell. The equivalent spring, damper, and piezoelectric patch form a resonator. Among them, the stiffness coefficient is $k_{r, i}\left(i=1,2, \ldots, a^{2}\right)$, where subscript $i$ represents the $i$ th resonator, and the damping coefficient can be introduced by means of a complex stiffness coefficient constant using the symbol $k_{i}$ to represent it, and it satisfies the relationship: $k_{i}=k_{r, i}\left(1+i \eta_{r, i}\right)$, where $\eta_{r, i}$ is the loss factor. Therefore, the flexible connection unit can be finally equivalent to the structure shown in Figure 3(b). In addition, an external shunt circuit is connected to the piezoelectric patch of a unit cell as shown in Figure 3(b); its impedance is $Z_{i}$.

To achieve the equivalent surface mass density and equivalent dynamic bending stiffness of the top and bottom facesheets of one period, it is based on EM method and can be completed by three steps. The first step is to determine the effective material parameters of a single piezoelectric patch with external shunt circuit. The second step is to derive the effective material parameters of regions $m$ and $s$ of the unit cell. And the last step is to determine the effective material parameters of the top and bottom periodic facesheet. 


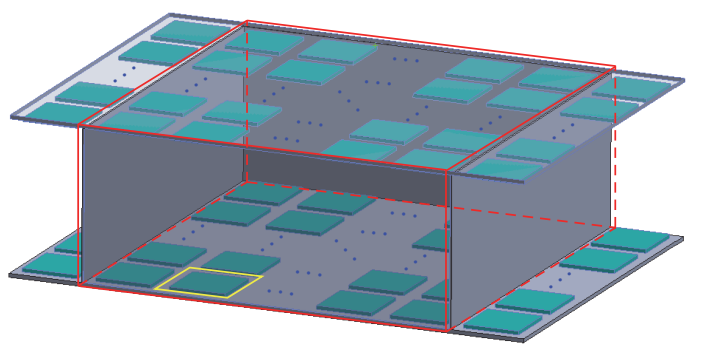

(a)

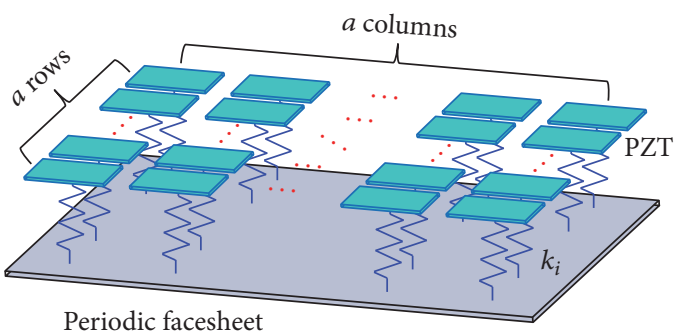

(b)

FIGURE 2: Local type view of basic element: (a) a periodic element (front rib-stiffener and top facesheet are transparent) and (b) flexible connections between piezoelectric patches and bottom facesheet.

Assuming that the displacement of each section of the spring has a linear distribution, the equivalent mass of the individual resonator can be obtained as follows:

$$
m_{r, i}=\frac{\rho_{p, i} h_{p, i} A_{p, i}+(1 / 3) m_{i}}{1-\omega^{2} / \omega_{r, i}^{2}\left(1+i \eta_{r, i}\right)},
$$

where $\rho_{p, i}, h_{p, i}$, and $A_{p, i}$, respectively, denote the volume mass density, thickness, and area of the shunted piezoelectric patch of the $i$ th resonator, $\omega=2 \pi f$ is the angular frequency, $m_{i}$ is the mass of the $i$ th equivalent spring, and $\omega_{r, i}=2 f_{r, i}$ represents the angular frequency of $i$ th resonator corresponding to the resonance frequency

$$
f_{r, i}=\frac{1}{2 \pi} \sqrt{\frac{k_{i}}{\rho_{p, i} h_{p, i} A_{p, i}+(1 / 3) m_{i}}} .
$$

Note that $m_{i} \ll \rho_{p} h_{p} A_{p}$; it can be regarded as a massless spring.

In addition, the piezoelectric patch of $i$ th resonator can be equivalent to an isotropic plate, whose equivalent Young's modulus $E_{p, i}$ and Poisson ratio $v_{p, i}$ are, respectively, as follows:

$$
\begin{gathered}
E_{p, i}=\frac{h_{p, i}\left(1+s Z_{i} C_{p, i}\right)}{h_{p, i} s_{11, i}^{E}\left(1+s Z C_{p, i}\right)-s Z_{i} d_{31, i}^{2} A_{p, i}}, \\
v_{p, i}=-\frac{s_{12, i}^{E}\left(1+s Z_{i} C_{P, i}\right)-s Z_{i} d_{31, i}^{2} A_{p, i} h_{p, i}^{-1}}{s_{11, i}^{E}\left(1+s Z_{i} C_{P, i}\right)-s Z_{i} d_{31, i}^{2} A_{p, i} h_{p, i}^{-1}},
\end{gathered}
$$

where $s=j \omega$ is the Laplace operator $(j=\sqrt{-1})$ and $s_{11, i}^{E}$ and $d_{31, i}$ are, respectively, the $i$ th piezoelectric patch compliance coefficient and piezoelectric constant. Subscripts 1, 2, and 3 are the three components of the corresponding parameter; its vector points along the direction of the three coordinate axes. $C_{p, i}$ is the inherent capacitance of $i$ th piezoelectric patch at constant stress, which can be expressed as

$$
C_{p, i}=\frac{A_{p, i} \varepsilon_{33, i}^{T}}{h_{p, i}}
$$

where $\varepsilon_{33, i}^{T}$ is the dielectric constant of the $i$ th piezoelectric patch at constant stress.

Therefore, the equivalent surface density and equivalent dynamic bending stiffness of regions $m$ and $s$ of the periodic facesheet can be obtained as

$$
\begin{aligned}
& \sigma_{j}(x, y)= \begin{cases}\sigma_{m, j}=\rho_{b, j} h_{b, j}+\sum_{i=1}^{a^{2}} \frac{m_{r, i}}{A_{p, i}}, & (x, y) \in m \\
\sigma_{s, j}=\rho_{b, j} h_{b, j}, & (x, y) \in s,\end{cases} \\
& D_{j}(x, y)= \begin{cases}D_{m, j}=\frac{E_{b, j} h_{b, j}^{3}}{12\left(1-v_{b, j}^{2}\right)}+\sum_{i=1}^{a^{2}} \frac{E_{p, i}\left[\left(h_{b, j}+2 h_{p, i}\right)^{3}-h_{b, j}^{3}\right]}{24\left(1-v_{p, i}^{2}\right)} \frac{1}{1-\omega^{2} / \omega_{r, i}^{2}\left(1+i \eta_{r, i}\right)}, \quad(x, y) \in m \\
D_{s, j}=\frac{E_{b, j} h_{b, j}^{3}}{12\left(1-v_{b, j}^{2}\right)}, & (x, y) \in s,\end{cases}
\end{aligned}
$$

where subscripts $j=1$ and 2 represent the top periodic facesheet and bottom periodic facesheet. $\rho_{b, j}, h_{b, j}, E_{b, j}$, and $v_{b, j}$ are, respectively, the volume mass density, thickness, Young's modulus, and Poisson ratio of the basic material of facesheet. 


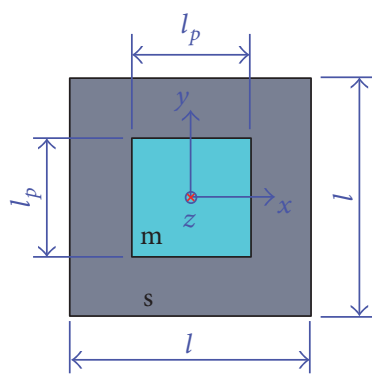

(a)

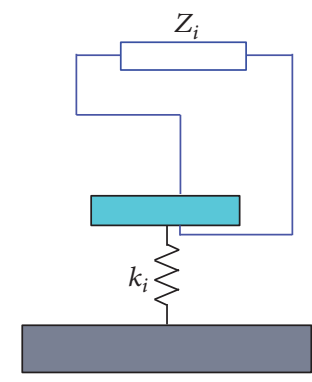

(b)
FIGURE 3: Unit cell of periodic subwavelength structure: (a) top view and (b) equivalent flexible connection and piezoelectric shunt circuit.

Furthermore, the expressions of the equivalent surface mass density and equivalent dynamic bending stiffness of the periodic facesheets are obtained by EM method as follows [37]:

$$
\begin{aligned}
\sigma_{\mathrm{eq}, j} & =\alpha \sigma_{m, j}+(1-\alpha) \sigma_{s, j}, \\
D_{\mathrm{eq}, j} & =\frac{D_{m, j} D_{s, j}}{(1-\alpha) D_{m, j}+\alpha D_{s, j}},
\end{aligned}
$$

where $\alpha$ denotes the ratio between the total area of region $m$ and the total area of region $s$ in one periodic facesheet, given by

$$
\alpha=\frac{l_{p}^{2}}{l^{2}}
$$

2.3. Dynamic Motions of Periodic Facesheets and Rib-Stiffeners. To a two-dimensional sandwich structure, reinforced by an orthogonally rib-stiffened core repeats in the $x$ - and $y$ direction and excited by a harmonic plane sound wave, the top and bottom facesheet responses can be expressed using space harmonic expansion as follows [10, 23, 38]:

$$
\begin{aligned}
w_{j} & (x, y, t) \\
= & \sum_{m=-\infty}^{+\infty} \sum_{n=-\infty}^{+\infty} \alpha_{j, m n} e^{-i\left[\left(k_{x}+2 m \pi / l_{x}\right) x+\left(k_{y}+2 n \pi / l_{y}\right) y-\omega t\right]},
\end{aligned}
$$

where subscripts $j=1$ and 2 also represent the top periodic facesheet and bottom periodic facesheet; the $(m, n)$ th harmonic wave components in the top and bottom facesheets have the same wavenumbers $\left(k_{x}+2 m \pi / l_{x}, k_{y}+2 n \pi / l_{y}\right)$ but different amplitudes: that is,

$$
\begin{aligned}
& \alpha_{j, m n}=\frac{1}{l_{x} l_{y}} \int_{0}^{l_{x}} \int_{0}^{l_{y}} w_{j}(x, y, t) \\
& \cdot e^{i\left[\left(k_{x}+2 m \pi / l_{x}\right) x+\left(k_{y}+2 m \pi / l_{y}\right) y-\omega t\right]} d x d y .
\end{aligned}
$$

Note that the wavenumbers $k_{x}+2 m \pi / l_{x}>0, k_{y}+2 n \pi / l_{y}>$ 0 denote positive-going harmonic waves in the $x$ - and $y$ direction and vice versa.
The orthogonal rib-stiffeners impose a strong constraint on the motions of the facesheets and then affect the sound transmission and wave propagation, where the influences of rib-stiffeners exist in the form of tensional forces, bending moments, and torsional moments, which satisfy the following expressions: tensional forces $=$ general force + inertial force, bending moments $=$ general bending moment + inertial bending moment, and torsional moments = general torsional moment + inertial torsional moment. The impacts at the interfaces between the top facesheet and $x / y$-wise ribstiffeners are marked by $\left(Q_{x}^{+}, M_{x}^{+}, M_{T x}^{+}\right)$and $\left(Q_{y}^{+}, M_{y}^{+}, M_{T y}^{+}\right)$ as shown in Figures 4(a) and 4(b), respectively. Similarly, the impacts at the interfaces between the bottom facesheet and $x / y$-wise rib-stiffeners are, respectively, marked by $\left(Q_{x}^{-}, M_{x}^{-}\right.$, $\left.M_{T x}^{-}\right)$and $\left(Q_{y}^{-}, M_{y}^{-}, M_{T y}^{-}\right)$as shown in Figure 5.

Taking all the impacts of the rib-stiffeners into consideration, and applying Hooke's law and the Newton's second law, the expressions of the tensional forces, bending moments, and torsional moments can be obtained as follows [10]:

(1) Tensional forces:

$$
\begin{aligned}
Q_{x}^{+} & =\sum_{m=-\infty}^{+\infty} \sum_{n=-\infty}^{+\infty}\left(-R_{\mathrm{Q} 1} \alpha_{1, m n}+R_{\mathrm{Q} 2} \alpha_{2, m n}\right) \\
\cdot & e^{-i\left[\left(k_{x}+2 m \pi / l_{x}\right) x+\left(k_{y}+2 m \pi / l_{y}\right) y\right]}, \\
Q_{x}^{-} & =\sum_{m=-\infty}^{+\infty} \sum_{n=-\infty}^{+\infty}\left(-R_{\mathrm{Q} 2} \alpha_{1, m n}+R_{\mathrm{Q} 1} \alpha_{2, m n}\right) \\
\cdot & e^{-i\left[\left(k_{x}+2 m \pi / l_{x}\right) x+\left(k_{y}+2 m \pi / l_{y}\right) y\right]}, \\
Q_{y}^{+} & =\sum_{m=-\infty}^{+\infty} \sum_{n=-\infty}^{+\infty}\left(-R_{\mathrm{Q} 3} \alpha_{1, m n}+R_{\mathrm{Q} 4} \alpha_{2, m n}\right) \\
\cdot & e^{-i\left[\left(k_{x}+2 m \pi / l_{x}\right) x+\left(k_{y}+2 m \pi / l_{y}\right) y\right]}, \\
Q_{y}^{-} & =\sum_{m=-\infty}^{+\infty} \sum_{n=-\infty}^{+\infty}\left(-R_{\mathrm{Q} 4} \alpha_{1, m n}+R_{\mathrm{Q} 3} \alpha_{2, m n}\right) \\
& \cdot e^{-i\left[\left(k_{x}+2 m \pi / l_{x}\right) x+\left(k_{y}+2 m \pi / l_{y}\right) y\right]} .
\end{aligned}
$$

(2) Bending moments:

$$
\begin{gathered}
M_{x}^{+}=\sum_{m=-\infty}^{+\infty} \sum_{n=-\infty}^{+\infty}\left(-R_{M 1} \alpha_{1, m n}+R_{M 2} \alpha_{2, m n}\right) \\
\cdot \alpha_{m}^{2} e^{-i\left[\left(k_{x}+2 m \pi / l_{x}\right) x+\left(k_{y}+2 n \pi / l_{y}\right) y\right]}, \\
M_{x}^{-}=\sum_{m=-\infty}^{+\infty} \sum_{n=-\infty}^{+\infty}\left(-R_{M 2} \alpha_{1, m n}+R_{M 1} \alpha_{2, m n}\right) \\
\cdot \alpha_{m}^{2} e^{-i\left[\left(k_{x}+2 m \pi / l_{x}\right) x+\left(k_{y}+2 n \pi / l_{y}\right) y\right]}, \\
M_{y}^{+}=\sum_{m=-\infty}^{+\infty} \sum_{n=-\infty}^{+\infty}\left(-R_{M 3} \alpha_{1, m n}+R_{M 4} \alpha_{2, m n}\right) \\
\cdot \beta_{n}^{2} e^{-i\left[\left(k_{x}+2 m \pi / l_{x}\right) x+\left(k_{y}+2 n \pi / l_{y}\right) y\right]},
\end{gathered}
$$




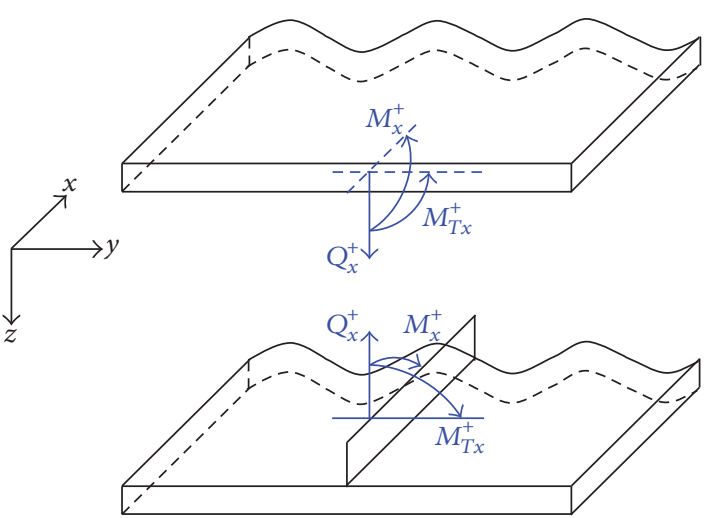

(a)

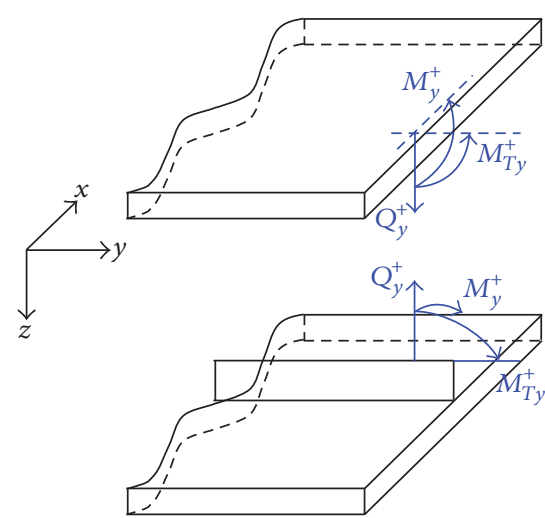

(b)

FIGURE 4: Convention for tensional forces, bending moments, and torsional moments between top facesheet and (a) $x$-wise and (b) $y$-wise stiffeners.

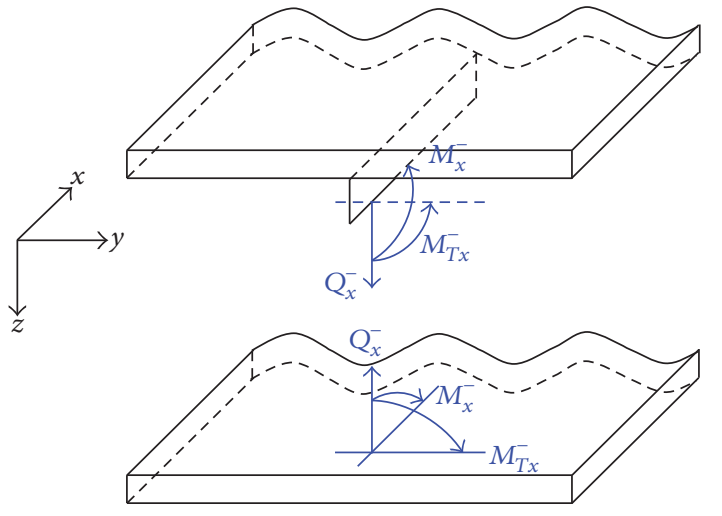

(a)

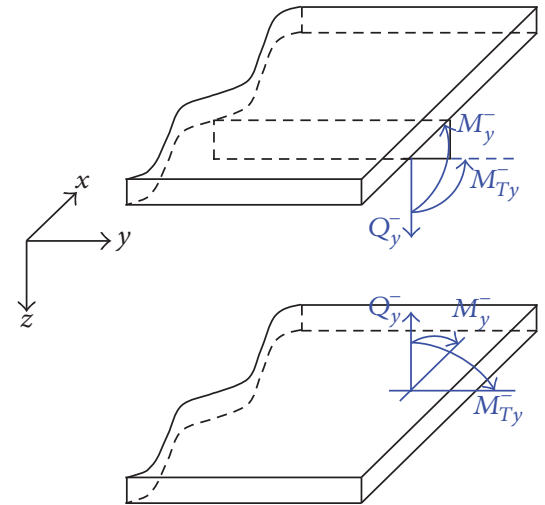

(b)

FIGURE 5: Convention for tensional forces, bending moments, and torsional moments between bottom facesheet and (a) $x$-wise and (b) $y$-wise stiffeners.

$$
\begin{aligned}
M_{y}^{-} & =\sum_{m=-\infty}^{+\infty} \sum_{n=-\infty}^{+\infty}\left(-R_{M 4} \alpha_{1, m n}+R_{M 3} \alpha_{2, m n}\right) \\
\cdot & \beta_{n}^{2} e^{-i\left[\left(k_{x}+2 m \pi / l_{x}\right) x+\left(k_{y}+2 n \pi / l_{y}\right) y\right]} .
\end{aligned}
$$

(3) Torsional moments:

$$
\begin{gathered}
M_{T x}^{+}=\sum_{m=-\infty}^{+\infty} \sum_{n=-\infty}^{+\infty}\left(-R_{T 1} \alpha_{1, m n}+R_{T 2} \alpha_{2, m n}\right) \\
\cdot \alpha_{m} \beta_{n} e^{-i\left[\left(k_{x}+2 m \pi / l_{x}\right)+\left(k_{y}+2 m \pi / l_{y}\right)\right]}, \\
M_{T x}^{-}=\sum_{m=-\infty}^{+\infty} \sum_{n=-\infty}^{+\infty}\left(-R_{T 2} \alpha_{1, m n}+R_{T 1} \alpha_{2, m n}\right) \\
\cdot \alpha_{m} \beta_{n} e^{-i\left[\left(k_{x}+2 m \pi / l_{x}\right)+\left(k_{y}+2 m \pi / l_{y}\right)\right]},
\end{gathered}
$$

$$
\begin{gathered}
M_{T y}^{+}=\sum_{m=-\infty}^{+\infty} \sum_{n=-\infty}^{+\infty}\left(-R_{T 3} \alpha_{1, m n}+R_{T 4} \alpha_{2, m n}\right) \\
\cdot \alpha_{m} \beta_{n} e^{-i\left[\left(k_{x}+2 m \pi / l_{x}\right)+\left(k_{y}+2 m \pi / l_{y}\right)\right]}, \\
M_{T y}^{-}=\sum_{m=-\infty}^{+\infty} \sum_{n=-\infty}^{+\infty}\left(-R_{T 4} \alpha_{1, m n}+R_{T 3} \alpha_{2, m n}\right) \\
\cdot \alpha_{m} \beta_{n} e^{-i\left[\left(k_{x}+2 m \pi / l_{x}\right)+\left(k_{y}+2 m \pi / l_{y}\right)\right]},
\end{gathered}
$$

where

$$
\begin{aligned}
& \alpha_{m}=k_{x}+\frac{2 m \pi}{l_{x}}, \\
& \beta_{n}=k_{y}+\frac{2 n \pi}{l_{y}} .
\end{aligned}
$$

In (12) to (14), $R_{\mathrm{Q} 1}, R_{\mathrm{Q} 2}, R_{\mathrm{Q} 3}$, and $R_{\mathrm{Q} 4}$ are tensional force coefficients, $R_{M 1}, R_{M 2}, R_{M 3}$, and $R_{M 4}$ are bending moment 
coefficients, and $R_{T 1}, R_{T 2}, R_{T 3}$, and $R_{T 4}$ are torsional moment coefficients. Their calculation expressions are as follows:

(1) Tensional force coefficients:

$$
\begin{aligned}
& R_{\mathrm{Q} 1}=\frac{K_{x}\left(K_{x}-\lambda_{x} \omega^{2}\right)}{2 K_{x}-\lambda_{x} \omega^{2}}, \\
& R_{\mathrm{Q} 2}=\frac{K_{x}^{2}}{2 K_{x}-\lambda_{x} \omega^{2}}, \\
& R_{\mathrm{Q} 3}=\frac{K_{y}\left(K_{y}-\lambda_{y} \omega^{2}\right)}{2 K_{y}-\lambda_{y} \omega^{2}}, \\
& R_{\mathrm{Q} 4}=\frac{K_{y}^{2}}{2 K_{y}-\lambda_{y} \omega^{2}} .
\end{aligned}
$$

(2) Bending moment coefficients:

$$
\begin{aligned}
& R_{M 1}=\frac{E_{x} I_{x}^{*}\left(E_{x} I_{x}^{*}-\rho_{x} I_{x} \omega^{2}\right)}{2 E_{x} I_{x}^{*}-\rho_{x} I_{x} \omega^{2}}, \\
& R_{M 2}=\frac{E_{x}^{2} I_{x}^{* 2}}{2 E_{x} I_{x}^{*}-\rho_{x} I_{x} \omega^{2}}, \\
& R_{M 3}=\frac{E_{y} I_{y}^{*}\left(E_{y} I_{y}^{*}-\rho_{y} I_{y} \omega^{2}\right)}{2 E_{y} I_{y}^{*}-\rho_{y} I_{y} \omega^{2}}, \\
& R_{M 4}=\frac{E_{y}^{2} I_{y}^{* 2}}{2 E_{y} I_{y}^{*}-\rho_{y} I_{y} \omega^{2}} .
\end{aligned}
$$

(3) Torsional moment coefficients:

$$
\begin{aligned}
& R_{T 1}=\frac{G_{x} J_{x}^{*}\left(G_{x} J_{x}^{*}-\rho_{x} J_{x} \omega^{2}\right)}{2 G_{x} J_{x}^{*}-\rho_{x} J_{x} \omega^{2}}, \\
& R_{T 2}=\frac{G_{x}^{2} J_{x}^{* 2}}{2 G_{x} J_{x}^{*}-\rho_{x} J_{x} \omega^{2}}, \\
& R_{T 3}=\frac{G_{y} J_{y}^{*}\left(G_{y} J_{y}^{*}-\rho_{y} J_{y} \omega^{2}\right)}{2 G_{y} J_{y}^{*}-\rho_{y} J_{y} \omega^{2}}, \\
& R_{T 4}=\frac{G_{y}^{2} J_{y}^{* 2}}{2 G_{y} J_{y}^{*}-\rho_{y} J_{y} \omega^{2}},
\end{aligned}
$$

where $\left(K_{x}, K_{y}\right),\left(E_{x} I_{x}^{*}, E_{y} I_{y}^{*}\right)$, and $\left(G_{x} J_{x}^{*}, G_{y} J_{y}^{*}\right)$ are, respectively, the tensional, bending, and torsional stiffness of half rib-stiffeners per unit length. $\left(\lambda_{x}, \lambda_{y}\right)$ and $\left(\rho_{x}, \rho_{y}\right)$ are the line and volume mass density for the rib-stiffeners, respectively. $\left(I_{x}, I_{y}\right)$ and $\left(J_{x}, J_{y}\right)$ are polar and torsional moment of inertia of the rib-stiffeners, where subscripts $x$ and $y$ denote the corresponding orientations of the orthogonal rib-stiffeners.
The geometrical properties of orthogonal rib-stiffeners are given by $[10,11]$

$$
\begin{aligned}
& K_{x}=\frac{E_{x} t_{x}}{d / 2}, \\
& K_{y}=\frac{E_{y} t_{y}}{d / 2}, \\
& I_{x}^{*}=\frac{t_{x}(d / 2)^{3}}{12}, \\
& I_{y}^{*}=\frac{t_{y}(d / 2)^{3}}{12} \text {, } \\
& I_{x}=\frac{t_{x} d^{3}}{12}, \\
& I_{y}=\frac{t_{y} d^{3}}{12} \\
& J_{x}^{*}=\frac{t_{x}^{3} d}{2}\left[\frac{1}{3}-\frac{64}{\pi^{5}} \frac{2 t_{x}}{d} \sum_{n=1,3,5, \ldots}^{+\infty} \frac{\tanh \left(n \pi d / 4 t_{x}\right)}{n^{5}}\right] \text {, } \\
& J_{y}^{*}=\frac{t_{y}^{3} d}{2}\left[\frac{1}{3}-\frac{64}{\pi^{5}} \frac{2 t_{y}}{d} \sum_{n=1,3,5, \ldots}^{+\infty} \frac{\tanh \left(n \pi d / 4 t_{y}\right)}{n^{5}}\right] \text {, } \\
& J_{x}=t_{x}^{3} d\left[\frac{1}{3}-\frac{64}{\pi^{5}} \frac{t_{x}}{d} \sum_{n=1,3,5, \ldots}^{+\infty} \frac{\tanh \left(n \pi d / 2 t_{x}\right)}{n^{5}}\right] \text {, } \\
& J_{y}=t_{y}^{3} d\left[\frac{1}{3}-\frac{64}{\pi^{5}} \frac{t_{y}}{d} \sum_{n=1,3,5, \ldots}^{+\infty} \frac{\tanh \left(n \pi d / 2 t_{y}\right)}{n^{5}}\right] \text {. }
\end{aligned}
$$

2.4. Continuity Conditions. The sound pressure in the top, middle, and bottom sound field is marked by $P_{1}(x, y, z, t)$, $P_{2}(x, y, z, t)$, and $P_{3}(x, y, z, t)$, which can be expressed by space harmonic series as $[7,10,23]$

$$
\begin{aligned}
& P_{1}(x, y, z, t)=P_{0} e^{-i\left(k_{x} x+k_{y} y+k_{z} z-\omega t\right)} \\
& +\sum_{m=-\infty} \sum_{n=-\infty}^{+\infty} \beta_{m n} e^{-i\left[\left(k_{x}+2 m \pi / l_{x}\right) x+\left(k_{y}+2 n \pi / l_{y}\right) y-k_{z, m n}-\omega t\right]}, \\
& P_{2}(x, y, z, t) \\
& \quad=\sum_{m=-\infty}^{+\infty} \sum_{n=-\infty}^{+\infty} \varepsilon_{m n} e^{-i\left[\left(k_{x}+2 m \pi / l_{x}\right) x+\left(k_{y}+2 n \pi / l_{y}\right) y-k_{z, m n}-\omega t\right]} \\
& \quad+\sum_{m=-\infty}^{+\infty} \sum_{n=-\infty}^{+\infty} \zeta_{m n} e^{-i\left[\left(k_{x}+2 m \pi / l_{x}\right) x+\left(k_{y}+2 n \pi / l_{y}\right) y-k_{z, m n}-\omega t\right]}, \\
& P_{3}(x, y, z, t) \\
& \quad=\sum_{m=-\infty}^{+\infty} \sum_{n=-\infty}^{+\infty} \xi_{m n} e^{-i\left[\left(k_{x}+2 m \pi / l_{x}\right) x+\left(k_{y}+2 n \pi / l_{y}\right) y-k_{z, m n}-\omega t\right]},
\end{aligned}
$$


where $k_{z, m n}$ is the $(m, n)$ th space harmonic wavenumber in the $z$-direction, which can by calculated from $[6,39]$

$$
k_{z, m n}=\sqrt{k_{0}^{2}-\left(k_{x}+\frac{2 m \pi}{l_{x}}\right)^{2}-\left(k_{y}+\frac{2 n \pi}{l_{y}}\right)^{2}},
$$

when $k_{0}^{2}<\left(k_{x}+2 m \pi / l_{x}\right)^{2}+\left(k_{y}+2 n \pi / l_{y}\right)^{2}$, the corresponding pressure waves become evanescent, and then the expression is replaced by

$$
k_{z, m n}=i \sqrt{\left(k_{x}+\frac{2 m \pi}{l_{x}}\right)^{2}+\left(k_{y}+\frac{2 n \pi}{l_{y}}\right)^{2}-k_{0}^{2}} .
$$

Moreover, the sound pressures in the three sound fields meet the wave equation [40]:

$$
\left(\frac{\partial^{2}}{\partial x^{2}}+\frac{\partial^{2}}{\partial y^{2}}+\frac{\partial^{2}}{\partial z^{2}}+k_{0}^{2}\right) P_{i}=0, \quad(i=1,2,3) .
$$

The continuity condition of fluid-structure coupling is to ensure that the facesheet velocity is equal to the fluid velocity in the facesheet surface, which is based on the momentum equation and satisfies the relationships as follows [19, 41]:

$$
\begin{aligned}
\left.\frac{\partial P_{1}}{\partial z}\right|_{z=0} & =\omega^{2} \rho_{0} w_{1}, \\
\left.\frac{\partial P_{2}}{\partial z}\right|_{z=h_{1}} & =\omega^{2} \rho_{0} w_{1}, \\
\left.\frac{\partial P_{2}}{\partial z}\right|_{z=h_{1}+d} & =\omega^{2} \rho_{0} w_{2}, \\
\left.\frac{\partial P_{3}}{\partial z}\right|_{z=h_{1}+d+h_{2}} & =\omega^{2} \rho_{0} w_{2},
\end{aligned}
$$

where $\rho_{0}$ is the density of fluid field.

By substituting (10) and (20) into (24), the relevant coefficients can be obtained as

$$
\begin{aligned}
& \beta_{m n}= \begin{cases}P_{0}+\frac{\omega^{2} \rho_{0} \alpha_{1, m n}}{i k_{z, m n}}, \quad(m=0 \& n=0) \\
\frac{\omega^{2} \rho_{0} \alpha_{1, m n}}{i k_{z, m n}}, \quad(m \neq 0 \| n \neq 0),\end{cases} \\
& \varepsilon_{m n}=\frac{\omega^{2} \rho_{0}\left[\alpha_{1, m n} e^{i k_{z, m n}\left(h_{1}+d\right)}-\alpha_{2, m n} e^{i k_{z, m n} h_{1}}\right]}{2 k_{z, m n} \sin \left(k_{z, m n} d\right)}, \\
& \zeta_{m n}=\frac{\omega^{2} \rho_{0}\left[\alpha_{1, m n} e^{-i k_{z, m n}\left(h_{1}+d\right)}-\alpha_{2, m n} e^{-i k_{z, m n} h_{1}}\right]}{2 k_{z, m n} \sin \left(k_{z, m n} d\right)}, \\
& \xi_{m n}=-\frac{\omega^{2} \rho_{0} \alpha_{2, m n}}{i k_{z, m n}} e^{i k_{z, m n}\left(h_{1}+d+h_{2}\right)} .
\end{aligned}
$$

2.5. Separation of Facesheet Response Amplitudes. As can be seen from (25) to (28), the facesheet response amplitudes $\alpha_{1, m n}$ and $\alpha_{2, m n}$ determine the motions of the facesheets and rib-stiffeners. Based on one periodic element, the amplitudes $\alpha_{1, m n}$ and $\alpha_{2, m n}$ can be derived by applying the virtual work principle $[6,7,10,23]$. Therefore, the virtual placements of the top and bottom facesheets can be obtained as

$$
\delta w_{j}=\delta \alpha_{j, m n} e^{-i\left[\left(k_{x}+2 m \pi / l_{x}\right) x+\left(k_{y}+2 n \pi / l_{y}\right) y\right]},
$$

where $\delta(\cdot)$ is the Dirac delta function.

The equations governing the vibration responses of the periodic facesheets are given by

$$
\begin{aligned}
& D_{\mathrm{eq}, 1} \nabla^{4} w_{1}+\sigma_{\mathrm{eq}, 1} \frac{\partial^{2} w_{1}}{\partial t^{2}}-P_{1}(x, y, 0) \\
& \quad+P_{2}\left(x, y, h_{1}\right)=0 \\
& D_{\mathrm{eq}, 2} \nabla^{4} w_{2}+\sigma_{\mathrm{eq}, 2} \frac{\partial^{2} w_{2}}{\partial t^{2}}-P_{2}\left(x, y, h_{1}+d\right) \\
& \quad+P_{3}\left(x, y, h_{1}+d+h_{2}\right)=0 .
\end{aligned}
$$

Moreover, the virtual works of periodic facesheets and $x / y$-wise rib-stiffeners are shown as follows:

(1) Periodic facesheets:

$$
\begin{aligned}
& \delta \Pi_{p 1}=\int_{0}^{l_{x}} \int_{0}^{l_{y}}\left[D_{\mathrm{eq}, 1} \nabla^{4} w_{1}+\sigma_{\mathrm{eq}, 1} \frac{\partial^{2} w_{1}}{\partial t^{2}}-P_{1}(x, y, 0)\right. \\
& \left.+P_{2}\left(x, y, h_{1}\right)\right] \delta w_{1}^{*} d x d y, \\
& \delta \Pi_{p 2}=\int_{0}^{l_{x}} \int_{0}^{l_{y}}\left[D_{\mathrm{eq}, 2} \nabla^{4} w_{2}+\sigma_{\mathrm{eq}, 2} \frac{\partial^{2} w_{2}}{\partial t^{2}}\right. \\
& \left.-P_{2}\left(x, y, h_{1}+d\right)+P_{3}\left(x, y, h_{1}+d+h_{2}\right)\right] \\
& \cdot \delta w_{2}^{*} d x d y .
\end{aligned}
$$

(2) $x$-wise rib-stiffeners:

$$
\begin{array}{r}
\delta \Pi_{x 1}=-\int_{0}^{l_{x}}\left[Q_{x}^{+}(x, 0)+\frac{\partial}{\partial x} M_{x}^{+}(x, 0)\right. \\
\left.+\frac{\partial}{\partial y} M_{T x}^{+}(x, 0)\right] \delta \alpha_{1, k l} e^{i\left[\left(k_{x}+2 k \pi / l_{x}\right) x\right]}, \\
\delta \Pi_{x 2}=-\int_{0}^{l_{x}}\left[Q_{x}^{-}(x, 0)+\frac{\partial}{\partial x} M_{x}^{-}(x, 0)\right. \\
\left.+\frac{\partial}{\partial y} M_{T x}^{-}(x, 0)\right] \delta \alpha_{2, k l} e^{i\left[\left(k_{x}+2 k \pi / l_{x}\right) x\right]} .
\end{array}
$$


(3) $y$-wise rib-stiffeners:

$$
\begin{array}{r}
\delta \Pi_{y 1}=-\int_{0}^{l_{y}}\left[Q_{y}^{+}(0, y)+\frac{\partial}{\partial y} M_{y}^{+}(0, y)\right. \\
\left.+\frac{\partial}{\partial x} M_{T y}^{+}(0, y)\right] \delta \alpha_{1, k l} e^{i\left[\left(k_{y}+2 l \pi / l_{y}\right) y\right]}, \\
\delta \Pi_{y 2}=-\int_{0}^{l_{y}}\left[Q_{y}^{-}(0, y)+\frac{\partial}{\partial y} M_{y}^{-}(0, y)\right. \\
\left.+\frac{\partial}{\partial x} M_{T y}^{-}(0, y)\right] \delta \alpha_{2, k l} e^{i\left[\left(k_{y}+2 l \pi / l_{y}\right) y\right]},
\end{array}
$$

where $\alpha_{1, k l}$ and $\alpha_{2, k l}$ are the modal amplitudes and

$$
\begin{gathered}
\frac{\partial}{\partial y} M_{T x}^{ \pm}(x, y=0)=\left.\frac{\partial}{\partial y} M_{T x}^{ \pm}(x, y)\right|_{y=0}, \\
\frac{\partial}{\partial x} M_{T y}^{ \pm}(x=0, y)=\left.\frac{\partial}{\partial x} M_{T y}^{ \pm}(x, y)\right|_{x=0} .
\end{gathered}
$$

Finally, the virtual work principle satisfies the fact that

$$
\delta \Pi_{p r}+\delta \Pi_{x r}+\delta \Pi_{y r}=0, \quad(r=1,2) .
$$

Substituting (10) and (20) into (31)-(33), then putting the simplified results into (35), it can be derived that

$$
\begin{aligned}
& {\left[D_{\mathrm{eq}, 1}\left(\alpha_{k}^{2}+\beta_{l}^{2}\right)^{2}-\sigma_{\mathrm{eq}, 1} \omega^{2}-\frac{\omega^{2} \rho_{0}}{i k_{z, k l}}\right.} \\
& \left.+\frac{\omega^{2} \rho_{0} \cos \left(k_{z, k l} d\right)}{k_{z, k l} \sin \left(k_{z, k l} d\right)}\right] l_{x} l_{y} \alpha_{1, k l}-\frac{\omega^{2} \rho_{0}}{k_{z, k l} \sin \left(k_{z, k l} d\right)} \\
& +l_{x} l_{y} \alpha_{2, k l} \\
& +\sum_{n=-\infty}^{+\infty}\left[R_{\mathrm{Q} 1}-i \alpha_{k}^{3} R_{M 1}-i \beta_{l} \alpha_{k} \beta_{n} R_{T 1}\right] l_{x} \alpha_{1, k n} \\
& +\sum_{n=-\infty}^{+\infty}\left[-R_{\mathrm{Q} 2}+i \alpha_{k}^{3} R_{M 2}+i \beta_{l} \alpha_{k} \beta_{n} R_{T 2}\right] l_{x} \alpha_{2, k n} \\
& +\sum_{n=-\infty}^{+\infty}\left[R_{\mathrm{Q} 3}-i \beta_{l}^{3} R_{M 3}-i \alpha_{k} \alpha_{m} \beta_{l} R_{T 3}\right] l_{y} \alpha_{1, m l} \\
& +\sum_{n=-\infty}^{+\infty}\left[-R_{\mathrm{Q} 4}+i \beta_{l}^{3} R_{M 4}+i \alpha_{k} \alpha_{l} \beta_{l} R_{T 4}\right] l_{y} \alpha_{2, m l}
\end{aligned}
$$

$$
\begin{aligned}
& -\frac{\omega^{2} \rho_{0}}{k_{z, k l} \sin \left(k_{z, k l} d\right)} l_{x} l_{y} \alpha_{1, k l}+\left[D_{\mathrm{eq}, 2}\left(\alpha_{k}^{2}+\beta_{l}^{2}\right)^{2}\right. \\
& \left.-\sigma_{\mathrm{eq}, 2} \omega^{2}-\frac{\omega^{2} \rho_{0}}{i k_{z, k l}}+\frac{\omega^{2} \rho_{0} \cos \left(k_{z, k l} d\right)}{k_{z, k l} \sin \left(k_{z, k l} d\right)}\right] l_{x} l_{y} \alpha_{2, k l} \\
& +\sum_{n=-\infty}^{+\infty}\left[-R_{\mathrm{Q} 2}+i \alpha_{k}^{3} R_{M 2}+i \beta_{l} \alpha_{k} \beta_{n} R_{T 2}\right] l_{x} \alpha_{1, k n} \\
& +\sum_{n=-\infty}^{+\infty}\left[R_{\mathrm{Q} 1}-i \alpha_{k}^{3} R_{M 1}-i \beta_{l} \alpha_{k} \beta_{n} R_{T 1}\right] l_{x} \alpha_{2, k n} \\
& +\sum_{n=-\infty}^{+\infty}\left[-R_{\mathrm{Q} 4}+i \beta_{l}^{3} R_{M 4}+i \alpha_{k} \alpha_{l} \beta_{l} R_{T 4}\right] l_{y} \alpha_{1, m l} \\
& \quad+\sum_{n=-\infty}^{+\infty}\left[R_{\mathrm{Q} 3}-i \beta_{l}^{3} R_{M 3}-i \alpha_{k} \alpha_{m} \beta_{l} R_{T 3}\right] l_{y} \alpha_{2, m l}=0,
\end{aligned}
$$

where (36) describe an infinite set of coupled equations. The solution becomes convergent when the single mode number is greater than a constant, that is, the sum-index $(m, n)$ in the finite range of $m=-\widehat{k} \sim \widehat{k}$ and $n=-\hat{l} \sim \hat{l}$. Therefore, the simultaneous equations can be written as follows in matrix form of finite order (i.e., $2 K L$, where $K=2 \widehat{k}+1, L=2 \widehat{l}+1$ )

$$
\left[\begin{array}{cc}
T_{11} & T_{12} \\
T_{21} & T_{22}
\end{array}\right]_{2 K L \times 2 K L}\left\{\begin{array}{c}
\alpha_{1, k l} \\
\alpha_{2, k l}
\end{array}\right\}_{2 K L \times 1}=\left\{\begin{array}{c}
F_{k l} \\
0
\end{array}\right\}_{2 K L \times 1} .
$$

The detailed derivation of (37) is similar to that in the literature $[6,7,10,11]$.

2.6. Sound Transmission Loss. From the above it can be known that the coefficients $\alpha_{j, m n}(j=1,2)$ have been solved; then the remaining unknown coefficients $\beta_{m n}, \varepsilon_{m n}, \zeta_{m n}$, and $\xi_{m n}$ can be found by using (25) (28), respectively. The sound transmission coefficient can then be calculated as

$$
\tau(\varphi, \theta)=\frac{\sum_{m=-\infty}^{+\infty} \sum_{m=-\infty}^{+\infty}\left|\xi_{m n}\right|^{2} \operatorname{Re}\left(k_{z, m n}\right)}{\left|P_{0}\right|^{2} k_{z}},
$$

which is dependent on the elevation angle $\varphi$ and azimuth angle $\theta$. The diffuse-field transmission coefficient is finally calculated by integrating $\tau(\theta, \varphi)$ over $\theta$ and $\varphi$ :

$$
\bar{\tau}=\frac{\int_{0}^{2 \pi} \int_{0}^{\varphi_{\mathrm{lim}}} \tau(\varphi, \theta) \sin \varphi \cos \varphi d \varphi d \theta}{\int_{0}^{2 \pi} \int_{0}^{\varphi_{\lim }} \sin \varphi \cos \varphi d \varphi d \theta},
$$

where $\varphi_{\lim }=90^{\circ}$ for random incidence and $\varphi_{\lim } \approx 78^{\circ}$ for field incidence transmission.

The sound transmission loss (STL) may be defined as [6$8,10,11,39$ ]

$$
\mathrm{STL}=-10 \log _{10}(\tau),
$$

where $\tau$ is calculated from (38) or (39). 
TABLE 1: Properties of one periodic element.

\begin{tabular}{|c|c|c|c|c|c|}
\hline$\rho_{b}\left(\mathrm{~kg} / \mathrm{m}^{3}\right)$ & $v_{b}$ & $E_{b}(\mathrm{Gpa})$ & $l(\mathrm{~mm})$ & \multicolumn{2}{|c|}{$h_{b}(\mathrm{~mm})$} \\
\hline 2700 & 0.33 & 70 & 40 & \multicolumn{2}{|c|}{2} \\
\hline$\overline{\rho_{p}\left(\mathrm{~kg} / \mathrm{m}^{3}\right)}$ & $l_{p}(\mathrm{~mm})$ & $h_{p}(\mathrm{~mm})$ & $s_{11}^{E} /\left(\mathrm{m}^{2} / \mathrm{N}\right)$ & \multicolumn{2}{|c|}{$s_{12}^{E} /\left(\mathrm{m}^{2} / \mathrm{N}\right)$} \\
\hline 7500 & 30 & 1 & $16.5 \times 10^{-12}$ & \multicolumn{2}{|c|}{$-4.78 \times 10^{-12}$} \\
\hline$d_{31} /(\mathrm{C} / \mathrm{N})$ & $\varepsilon_{33}^{T} /(\mathrm{F} / \mathrm{m})$ & $f_{r, i}(\mathrm{~Hz})$ & $-C_{\mathrm{eq}}(\mathrm{F})$ & \multicolumn{2}{|c|}{$G_{x} \& G_{y}(\mathrm{Gpa})$} \\
\hline$-2.74 \times 10^{-10}$ & $3.01 \times 10^{-8}$ & $150+10(i-1)$ & $-0.3 C_{p}$ & \multicolumn{2}{|c|}{27} \\
\hline$\rho_{x} \& \rho_{y}\left(\mathrm{~kg} / \mathrm{m}^{3}\right)$ & $E_{x} \& E_{y}(\mathrm{Gpa})$ & $l_{x} \& l_{y}(\mathrm{~mm})$ & $t_{x} \& t_{y}(\mathrm{~mm})$ & $\varphi\left(^{\circ}\right)$ & $\theta\left(^{\circ}\right)$ \\
\hline 2700 & 70 & 200 & 1 & 30 & 30 \\
\hline
\end{tabular}

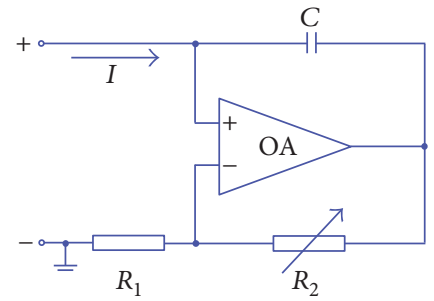

FIGURE 6: Equivalent negative-capacitance circuit.

\section{Fundamental Conditions of Research}

3.1. Basic Parameters. To the STL problem of the periodic sandwich structure as stated above, the geometrical and characteristic parameters have a direct influence on the sound insulation performance, and the STL performance of periodic structure can be characterized by one periodic element.

According to the periodic element as shown in Figure 2(a), the piezoelectric patches of each facesheet are selected as 25 pieces (i.e., $a=5$ ). The corresponding external shunt circuits are the equivalent negative-capacitance (NC) circuit as shown in Figure 6, which consists of a fixed resistor $\left(R_{1}\right)$, an adjustable resistor $\left(R_{2}\right)$, a capacitor $(C)$, and an operational amplifier (OA). Among them, the fixed resistor is composed of the internal resistor of piezoelectric patch and the external shunt resistor. The equivalent negative capacitance is given by

$$
-C_{\mathrm{eq}}=-\frac{R_{1}}{R_{2}} C .
$$

Therefore, the impedance of each external shunted circuit can be obtained as

$$
Z_{i}=\frac{1}{-C_{\mathrm{eq}} \cdot s} .
$$

In addition, the properties of the facesheet material (aluminum) and piezoelectric patch $(\mathrm{PZT}$ 5 $\mathrm{H})$ are represented in Table 1; the meaning of each symbol is as stated above.

3.2. Verification of Subwavelength Hypothesis. Since the EM theory is valid only in the subwavelength regime, it is

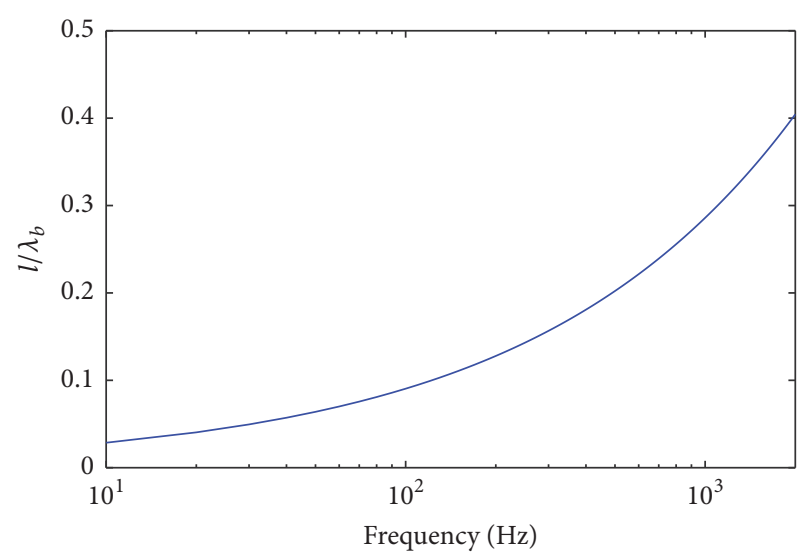

FIgure 7: The ratio $\left(l / \lambda_{b}\right)$ of the lattice constant to the flexural wavelength.

necessary to verify whether the unit cell in Figure 3(a) satisfies the subwavelength hypothesis, which requires that the lattice constant $(l)$ is smaller than the flexural wavelength $\left(\lambda_{b}\right)$ of the substrate, and $\lambda_{b}$ is defined by

$$
\lambda_{b}=2 \pi \sqrt[4]{\frac{D_{s}}{\rho_{b} h_{b} \omega^{2}}} .
$$

The ratio $\left(l / \lambda_{b}\right)$ of the lattice constant to the flexural wavelength is plotted in Figure 7 over the frequency range of 20-2000 Hz. Figure 7 describes that the ratio increases with frequency; that is, the calculation accuracy of the EM model decreases with the increasing frequency. It is can be seen that the ratio $l / \lambda_{b}$ is smaller than 0.5 and confirms the validity of the hypothesis.

3.3. Criteria for Convergence. For the research sandwich structure, there must be enough truncations about infinite $m$ and $n$ to ensure the convergence and accuracy of the solution, but when the number of terms is too large, it needs high hardware configuration and low computational efficiency as the cost. The description of a feasible criterion [6] is when the solution converges at a given frequency; it is convergent in the region below that frequency. Therefore, it only needs to determine 


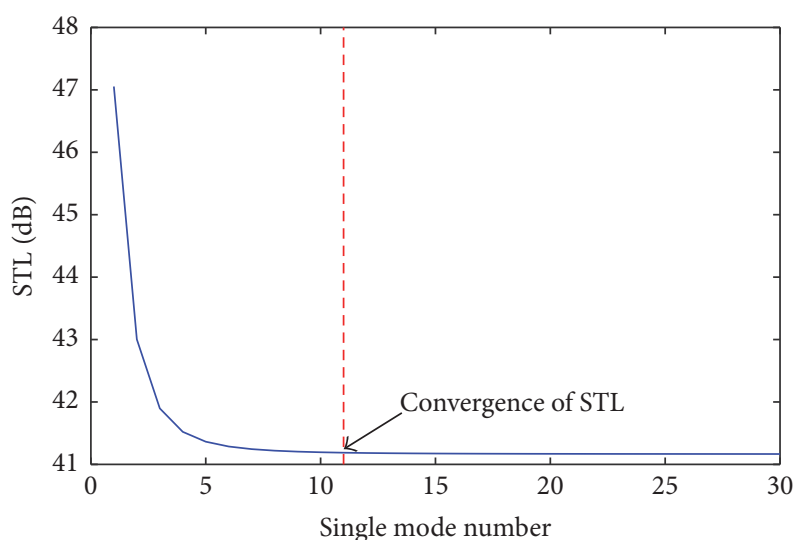

FIGURE 8: Convergence check of the infinite orthogonally ribstiffened sandwich structure at the highest frequency of $2 \mathrm{kHz}$.

the necessary number of terms corresponding to the highest frequency (i.e., $2 \mathrm{kHz}$ ). Moreover, the convergence check of the sandwich structure is determined by the following three steps. The first step is to calculate the STL performance at the highest frequency. The second step is to add one term to further calculate the corresponding STL. In the last step, in order to find whether the difference between the two results is within the preset error band $(0.008 \mathrm{~dB}$ in this work), the solution can be considered convergent and the corresponding number of terms is suitable for calculating the STL performances at all other frequencies below $2 \mathrm{kHz}$.

Since the infinite orthogonally rib-stiffened sandwich structure is symmetrical about $x$ - and $y$-axis, all the equations have been evolved into a finite set of equations with $m=$ $-\widehat{k} \sim \widehat{k}$ and $n=-\hat{l} \sim \widehat{l}$, and an assumption have been made where $\hat{k}=\widehat{l}$. Figure 8 illustrates that the STL decreases gradually with the single mode number $(\hat{k}$ or $\widehat{l})$ increasing at the highest frequency of $2 \mathrm{kHz}$, and finally it tends to be stable. In addition, the results of Figure 8 show that the solution achieved convergence conditions when single mode number $\widehat{k}$ (or $\widehat{l}$ ) $\geq 11$; that is, it requires at least 529 terms to ensure solution convergence.

\section{Calculation of Sound Transmission Loss}

4.1. Accuracy of the Theoretical Model. To represent the accuracy of the theoretical model, we compare the theoretical values with the finite element simulation results based on VA One software and the reference experimental data is this section, respectively.

4.1.1. Simulation Comparison. For a theoretical model, its feasibility and correctness are the key to development. In this section, the finite element analysis of the research structure is carried out by using VA One, vibroacoustics simulation software developed by the ESI Group. The finite element results are then compared with the theoretical values to verify the accuracy of the theoretical model.

Since VA One can perform finite element simulation of periodic subsystems, it is only necessary to analyze the periodic element in Figure 2(a). For the orthogonally ribstiffened sandwich structure with no shunt piezoelectric patches, the corresponding finite element model is shown in Figure 9(a), which includes a periodic element, two semiinfinite fluids (SIFs), and a diffuse acoustic field (DAF). Consider the finite periodic element with fluid on both sides, the acoustic transmission coefficient through the structure can be derived by the diffuse-field reciprocity result [42]. For an eigensolution associated with a pair of phase constants $\left(\varepsilon_{x}, \varepsilon_{y}\right)$, the radiation stiffness in the SIF on side $i$ is written as follows:

$$
\begin{aligned}
& D_{\text {rad }}^{(i)}\left(\varepsilon_{x}, \varepsilon_{y}, \omega\right)=\frac{1}{\pi^{2}} \int_{-\infty}^{\infty} \int_{-\infty}^{\infty}\left|\widetilde{\Phi}_{\varepsilon_{x}, \varepsilon_{y}}\left(k_{x}, k_{y}\right)\right|^{2} \\
& \cdot G_{i}^{*}\left(k_{x}, k_{y}, \omega\right) d k_{x} d k_{y},
\end{aligned}
$$

where $G_{i}$ is the spatial Fourier transform of the freefield Green function [43] and $\widetilde{\Phi}_{\varepsilon_{x}, \varepsilon_{y}}$ is the spatial Fourier transform of displacement field over the entire periodic structure (obtained from the spatial Fourier transform of the eigenvector over a single research element). When $k_{1}$ denotes the acoustic wavenumber in the source cavity and $A$ is the surface area per periodic element coupled to the fluid on the source side, the transmission loss is obtained from the diffuse-field reciprocity result given by the following equation:

$$
\tau(\omega)=\frac{4 \pi}{A k_{1}^{2}} \sum_{n}\left\langle\frac{\operatorname{Im}\left\{D_{\mathrm{rad}}^{(1)}\left(\varepsilon_{x}, \varepsilon_{y}, \omega\right)\right\} \operatorname{Im}\left\{D_{\mathrm{rad}}^{(2)}\left(\varepsilon_{x}, \varepsilon_{y}, \omega\right)\right\}}{\left|2 N_{x} N_{y}\left(\Omega\left(\varepsilon_{x}, \varepsilon_{y}\right)^{2}(1+i \eta)-\omega^{2}\right)+\left(D_{\mathrm{rad}}^{(1)}\left(\varepsilon_{x}, \varepsilon_{y}, \omega\right) / 2\right)+\left(D_{\mathrm{rad}}^{(2)}\left(\varepsilon_{x}, \varepsilon_{y}, \omega\right) / 2\right)\right|}\right\rangle,
$$

where the sum runs over the eigensolutions associated with the phase constants pair $\left(\varepsilon_{x}, \varepsilon_{y}\right)$ and $\operatorname{Im}\{\}$ denotes the imaginary part. For a given frequency band $\Delta \omega$, the contribution to the transmission from the modes with natural frequency within the band (i.e., $\Omega\left(\varepsilon_{x}, \varepsilon_{y}\right) \in \Delta \omega$ ) defines the resonant transmission and is analytically averaged over the band.
Alternatively, the nonresonant transmission is obtained from the contribution of the modes with natural frequency below and above the frequency band.

For the finite element model, it requires that the minimum wavelength corresponding to the highest frequency contains at least four grids. The geometrical and material 


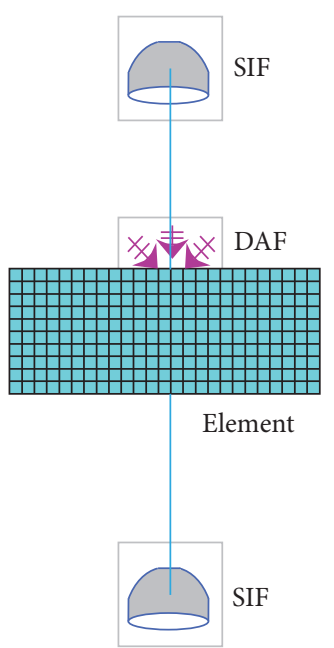

(a)

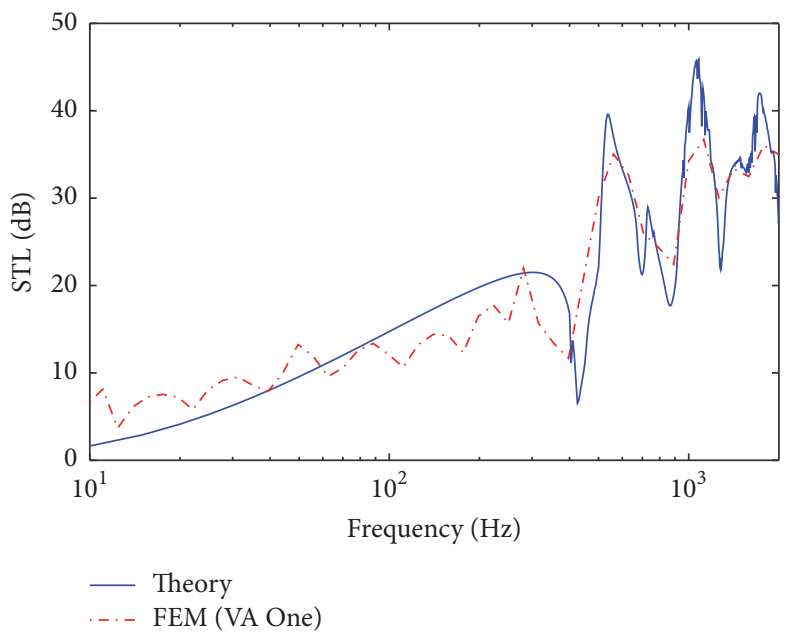

(b)

FIGURE 9: Comparison of the theoretical prediction values and simulation results for the orthogonally rib-stiffened sandwich structure with no shunt piezoelectric patches: (a) FE model and (b) STLs.

parameters of the periodic element and the fluid properties are shown in Table 1 . To improve the solving efficiency, the finite element model is used, 1/6 octave in the frequency range of $10 \mathrm{~Hz}-2000 \mathrm{~Hz}$. Furthermore, the simulation results obtained by the finite element method (FEM) are compared with the theoretical values obtained by integration when $\varphi_{\text {lim }}$ is limited to $78^{\circ}$ as shown in Figure 9(b).

As can be seen from Figure 9(b), the STL curve simulated by the FEM has a similar trend with the theoretical calculation curve. Although the simulation curve is not as smooth as the theoretical curve, the corresponding valley frequencies (such as around $425 \mathrm{~Hz}, 865 \mathrm{~Hz}$, and $1275 \mathrm{~Hz}$ ) and peak frequencies (such as around $555 \mathrm{~Hz}, 1115 \mathrm{~Hz}$, and $1725 \mathrm{~Hz}$ ) can effectively match. In addition, the difference between the two STL curves is within acceptable limits. Therefore, the theoretical model established for the orthogonally ribstiffened sandwich structure can predict the sound insulation.

4.1.2. Cited Experimental Comparison. The feasibility and accuracy of the theoretical model can also be verified by a comparison, between the theoretical prediction values and the experimental results in the internal report IRCIR-761 [24], completed by National Research Council of Canada (NRCC) in March 1998. The experimental specimen of number G16_SS90(610)_G16 is made up from two parallel gypsum boards, which are separated by an air cavity and linked with periodically distributed "C-section" steel studs. For this reason, the research sandwich structure is simplified as a typically infinite sandwich structure with unidirectionally parallel rib-stiffeners; that is, the theoretical model is not restricted by the subwavelength assumption. In addition, the material and geometrical properties of the structure adopted by the experiment G16_SS90(610)_G16 are completely followed in the comparison.

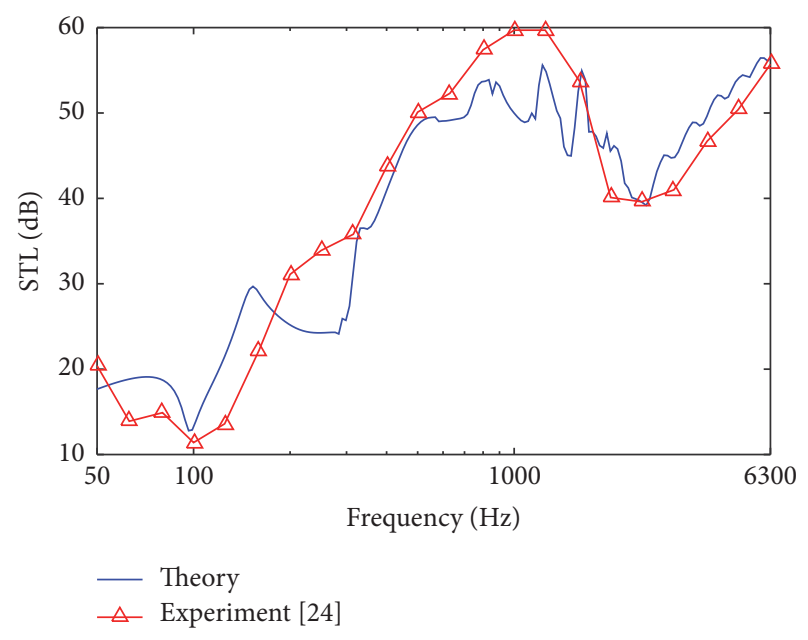

FIGURE 10: Comparison of the theoretical prediction values and experimental results for the typical sandwich structure with unidirectionally parallel rib-stiffeners.

Figure 10 shows a comparison of the predictions from theoretical model and test data from internal report IRC-IR761 [24]. Among them, the STL measurements are taken in $1 / 3$ octave bands from $50 \mathrm{~Hz}$ to $6300 \mathrm{~Hz}$, and the STL is calculated in $1 / 27$ octave bands with a diffuse-field integration $\operatorname{limit} \varphi_{\lim }$ of $78^{\circ}$ in the same frequency range.

It can be seen from Figure 10 that overall the comparison of theoretical prediction values and experimental results is satisfactory, except for a certain discrepancy in narrowband $200 \mathrm{~Hz}-300 \mathrm{~Hz}$ and in the vicinity of $1000 \mathrm{~Hz}$. Since the influence factors considered in the theoretical model are not comprehensive in the experimental measurement, the theory and experiment must be more or less different. As 


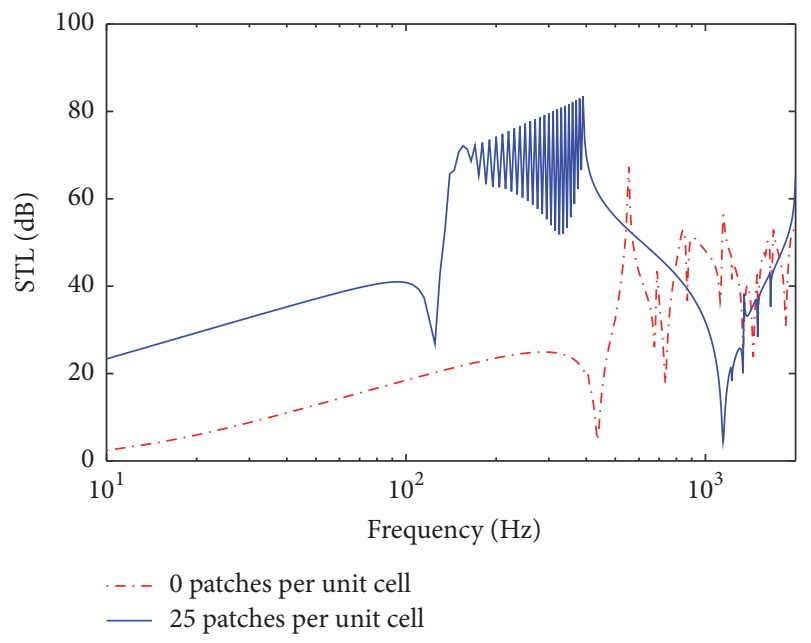

FIGURE 11: Comparison of the STL between the contrast structure with no piezoelectric patches and the research sandwich structure is flexibly connected with 25 piezoelectric patches in each unit cell.

Hongisto et al. [28] did the STL experiments on nine steel passage doors and nine timber passage doors using the sound intensity method, the average difference between the results and the corresponding theory is in the range of -3 to $12 \mathrm{~dB}$. Therefore, the comparison shows that the theoretical model can be used to analyze the structure characteristics and acoustical properties of the sandwich structure with ribstiffeners as a core accurately.

4.2. Validity of Theoretical Model. To explore the validity of the research sandwich structure composed of 25 piezoelectric patches in each unit cell, the orthogonally rib-stiffened sandwich structure with no shunt piezoelectric patches is introduced as a contrast model. The material parameters and geometrical properties of the two calculation models are equal as shown in Table 1 . Since the equivalent facesheet parameters of each model are identical, the natural frequencies of sandwich structure with rib-stiffened core can be calculated by the following formula:

$$
\begin{aligned}
& f_{\text {nat }} \\
& =\sqrt{\frac{D}{\sigma} \frac{\left[\left(m \pi / l_{x}\right)^{2}+\left(n \pi / l_{y}\right)^{2}\right]^{2}}{1+(D / C)\left(n \pi / l_{y}\right)^{2} /\left(1+((1-v) / 2)(D / C)\left(m \pi / l_{x}\right)^{2}\right)}},
\end{aligned}
$$

where $D=D_{\mathrm{eq}, j}(j=1$ or 2$)$ and $v$ are, respectively, the equivalent dynamic bending stiffness and Poisson's ratio of the facesheet. $\sigma$ is the total equivalent surface density of the studied structure. $C$ is shear stiffness of the rib-stiffener.

The STLs of the two calculation models are compared in the frequency range $10 \mathrm{~Hz}-2000 \mathrm{~Hz}$ as shown in Figure 11. It illustrates that the STL of the research structure is better than that of the contrast structure in the whole frequency band, except for the vicinity of the second valley frequency $1146.9 \mathrm{~Hz}$, which is generated by the $(3,0)$-order modal of

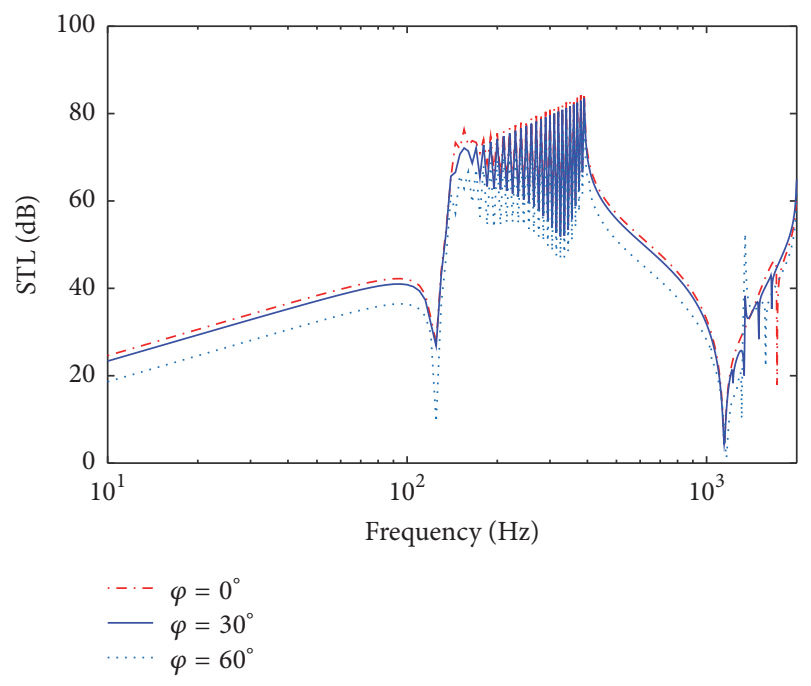

FIGURE 12: STLs of the research structure under the condition of having selected elevation angles.

the research structure. More concretely, as the number of piezoelectric patches on the top and bottom facesheet in a typically periodic element increases from 0 to 25 , the increment of STL exceeds $20 \mathrm{~dB}$ over the frequency range $10 \mathrm{~Hz}-120 \mathrm{~Hz}$. Moreover, the first valley frequency is moved from $437.3 \mathrm{~Hz}$ to $133.1 \mathrm{~Hz}$; it corresponds to the $(1,5)$-order modal of the contrast structure and the $(1,1)$-order modal of the research structure, respectively. In addition, it can be concluded from Figure 11 that the multimode resonant frequencies are generated by the resonators, which are formed by the flexible connections and the corresponding piezoelectric patches; they can improve the STL effectively and broaden the sound insulation bandwidth of the research structure.

\subsection{Parameter Studies}

4.3.1. Effect of Elevation Angle. According to the research structure, the azimuth angle has no influence on the STL in the whole frequency band when the elevation angle is certain. Therefore, only the effect of the elevation angle on the STL is studied under constant azimuth angle in the frequency range of $10 \mathrm{~Hz}-2000 \mathrm{~Hz}$.

Under the condition of the azimuth angle being identica 1 to $30^{\circ}$ (i.e., $\theta=30^{\circ}$ ), the STLs calculated for three different elevation angles (i.e., $\varphi=0^{\circ}, 30^{\circ}$, and $60^{\circ}$ ) are plotted in Figure 12, which illustrates that the STL decreases with increasing elevation angle due to the waveform conversion. There is no waveform conversion when $\varphi=0^{\circ}$ (i.e., normal incident), but with the increases of elevation angle (i.e., oblique incident) the waveform conversion occurs, resulting in the fact that the space harmonic wavenumber in $z$-direction changes, and finally the sound transmission coefficient increases as the elevation angle increases. Figure 12 also indicates that the first valley frequency is fixed at $133.1 \mathrm{~Hz}$; this phenomenon is independent of the incident angle and is determined by the inherent characteristics of the research structure. 


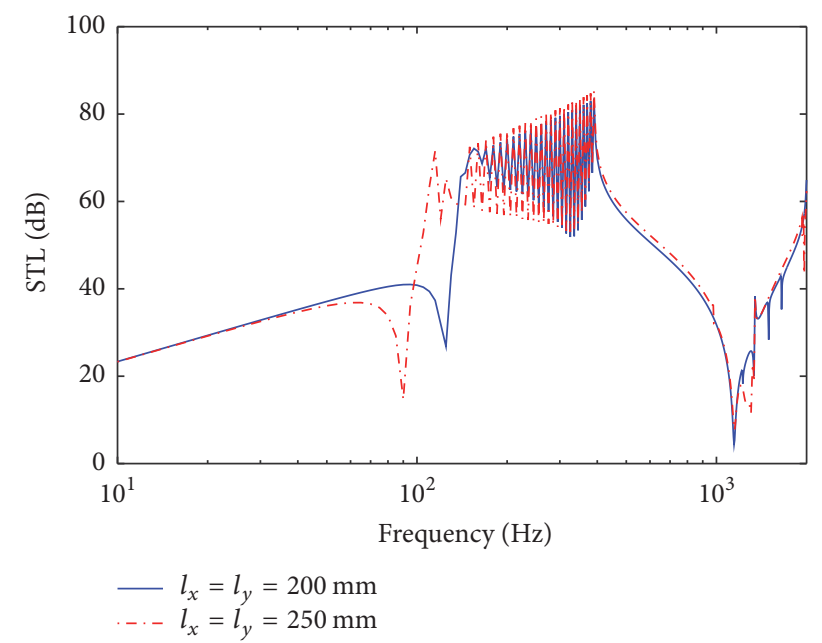

FIGURE 13: STLs of the research structure under the condition of having selecting rib-stiffener spacing.

4.3.2. Effect of Rib-Stiffener Spacing. Rib-stiffener spacing $l_{x}$ and spacing $l_{y}$ have a great impact on the properties of structure-borne and fluid-borne transmission. Therefore, on the basis that the ratio between the total area of region $\mathrm{m}$ and the total area of region $\mathrm{s}$ in one periodic facesheet is fixed at 9/16, the rib-stiffener spacing of two different studied sandwich structures is, respectively, selected as $l_{x}=l_{y}=$ $200 \mathrm{~mm}$ and $l_{x}=l_{y}=250 \mathrm{~mm}$. The STL curves are obtained as shown in Figure 13, wherein the group with spacing of $250 \mathrm{~mm}$ is a contrast structure, the lattice constant and width of piezoelectric patch are, respectively, $50 \mathrm{~mm}$ and $37.5 \mathrm{~mm}$, and the remaining parameters are unchanged.

As can be seen from Figure 13, the two STL curves have similar trends, but the first valley frequency of the contrast structure is lower than that of the research structure; the exact values are $87.0 \mathrm{~Hz}$ and $133.1 \mathrm{~Hz}$, respectively. Therefore, increasing the rib-stiffener spacing improves the STL and broadens sound transmission bandwidth to a certain extent. This is because the natural frequency decreases as the ribstiffener spacing increases, and the modal amplitude coefficient at the resonant frequency increases rapidly.

4.3.3. Effect of Facesheet Thickness. For the research structure, the increase of total mass to some extent can improve the STL performance, but the location of the valley frequencies limit the sound insulation and bandwidth of the structure, thereby reducing its engineering application value. Therefore, in order to minimize the total mass of the structure, the rational design of structural geometry to improve its sound insulation performance is critical. To quantify the effects of facesheet thickness, the three STL versus frequency curves of different studied sandwich structures under the condition of the thicknesses $\left(h_{1}, h_{2}\right)$, respectively, selected as $(1.0,1.0),(1.5$, $1.5)$, and $(2.0,2.0) \mathrm{mm}$, are presented in Figure 14.

Figure 14 indicates that, with the increase of the facesheet thicknesses $\left(h_{1}, h_{2}\right)$, the first valley frequency moves to a higher frequency, and the second valley frequency moves to

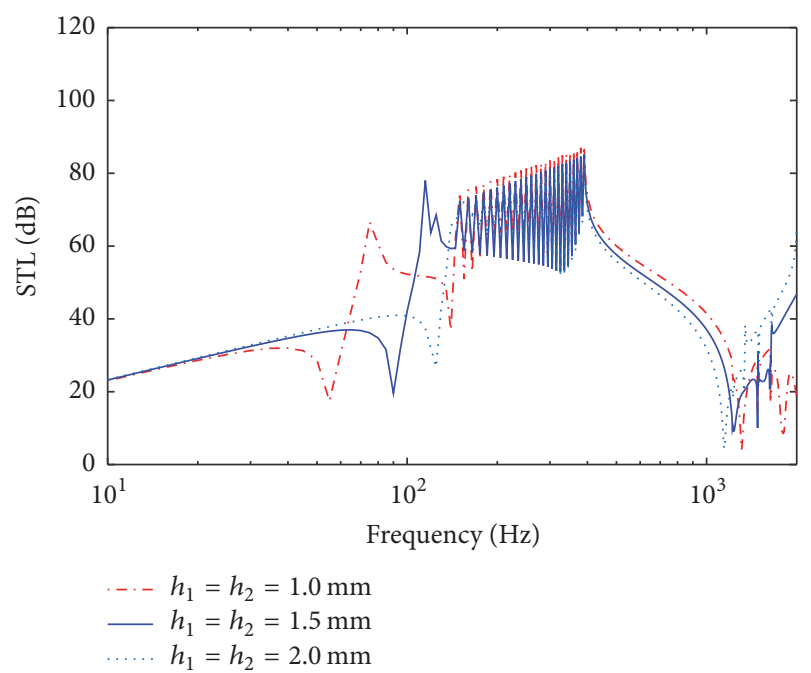

FIGURE 14: STLs of the research structure under the condition of having selecting facesheet thicknesses.

a lower frequency. That is, the sound insulation bandwidth gradually narrows, because the equivalent surface mass density and equivalent dynamic bending stiffness of the facesheet change as the thicknesses increase. For the three studied models with $(1.0,1.0),(1.5,1.5)$, and $(2.0,2.0) \mathrm{mm}$, the natural frequency is $57.4 \mathrm{~Hz}, 91.4 \mathrm{~Hz}$, and $133.1 \mathrm{~Hz}$, which are generated by the $(1,1)$-order modal of each model. And the second valley values were $1312.6 \mathrm{~Hz}, 1266.6 \mathrm{H}$, and $1146.9 \mathrm{~Hz}$, respectively, by the role of the $(5,7)-,(4,2)-$, and $(3,0)$ order modal. In addition, the increments of sound insulation caused by the equivalent resonators tend to deteriorate when the thicknesses $\left(h_{1}, h_{2}\right)$ decrease, since the multimode local resonance frequencies are close to other modal frequencies between the first valley frequency and the second valley frequency of the studied sandwich structure.

4.3.4. Effect of External Shunt Circuit. In view of the equivalent negative-capacitance circuit shown in Figure 6, the contrast curves depicted in Figure 15 are the STLs corresponding to the negative capacitance $-C_{\mathrm{eq}}$ of $-0.1 C_{p},-0.3 C_{p}$, and $-0.5 C_{p}$, respectively. This figure shows that, as the negative capacitance increases, the second valley frequency changes slightly, with the exact values being $1143.2 \mathrm{~Hz}, 1146.9 \mathrm{~Hz}$, and $1155.0 \mathrm{~Hz}$, respectively. In addition, the change in negative capacitance has no effect on the STL of the research sandwich structure in the range of less than about $1000 \mathrm{~Hz}$, but beyond this frequency, the amplitudes of the STLs and all extreme frequencies are affected. This is because the negative capacitance directly affects the equivalent Young's modulus and equivalent Poisson's ratio of the facesheets in the research structure.

\section{Conclusions}

This paper is related to the low-frequency STL of an infinite orthogonally rib-stiffened sandwich structure, with the inner sides of the upper and bottom facesheet flexibly connected 


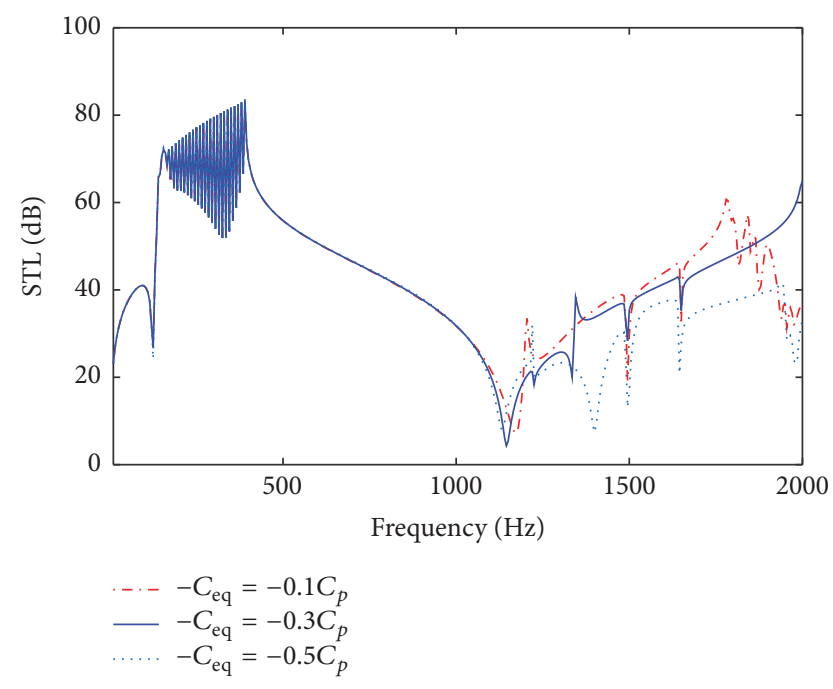

FIGURE 15: STLs of the research structure under the condition of having selecting negative capacitances.

with periodic subwavelength arrays of shunted piezoelectric patches. For exploring its low-frequency vibroacoustic characteristics, a complete theoretical model of STL for solving the research sandwich structure can be built in three steps. The first step is that the upper and bottom facesheet with periodic subwavelength arrays of shunted piezoelectric patches are equivalent to two homogeneous facesheets in a periodic element by effective medium (EM) method. The second step is to establish the dynamic motion governing equations by space harmonic method, and the tensional forces, bending moments, and torsional moments of the rib-stiffeners as well as the inertial terms are taken into account; then the amplitude coefficients of the equivalent facesheets are separated by the virtual work principle under the velocity continuity conditions. The third step is to deduce the calculation formula of STL. But most importantly, the application of the theoretical model has two prerequisites. One is that the unit cells in facesheets satisfy the subwavelength assumption; that is, the lattice constant is smaller than the flexural wavelength of the substrate. The other is that the infinite sets of coupled equations must be truncated to be finite by the convergence criterion; that is, at the highest frequency, the difference between the STLs corresponding to two adjacent terms is within the preset error band $(0.008 \mathrm{~dB}$ in this work).

To verify the validity and feasibility of the theoretical model, the finite element simulation of the orthogonally ribstiffened sandwich structure is carried out. The corresponding valley frequencies and peak frequencies can effectively match. Then the complete model is simplified to a typically infinite sandwich structure with unidirectionally parallel ribstiffeners and compares with the experimental results in the frequency range $50 \mathrm{~Hz}-6300 \mathrm{~Hz}$ of the experimental specimen numbered G16_SS90(610)_G16 in the internal report of the NRCC. The theoretical values are in good agreement with the experimental values, except for a certain discrepancy in narrowband $200 \mathrm{~Hz}-300 \mathrm{~Hz}$ and in the vicinity of $1000 \mathrm{~Hz}$.
Furthermore, the STL of the complete model is compared with that of the typical orthogonally rib-stiffened sandwich structure, which proves that the research sandwich structure has good sound insulation performance.

At the end of the work, the influences of the parameters such as the elevation angle, rib-stiffener spacing, facesheet thicknesses, and negative capacitance on the research structure are studied, and four main conclusions are drawn. First, the STL decreases with increasing elevation angle, and it has no effect on the bandwidth. Second, appropriately increasing the rib-stiffener spacing can improve the sound insulation and broaden the bandwidth to a certain extent. Third, as the thickness of the facesheets increases from $1 \mathrm{~mm}$ to $2 \mathrm{~mm}$, the sound insulation bandwidth gradually narrows, and the increments of sound insulation caused by the equivalent resonators become better. Finally, the change of negative capacitance has a slight influence on the bandwidth of STL, but it has a certain effect on the amplitude of STL above the second valley frequency.

\section{Conflicts of Interest}

The authors declare that they have no conflicts of interest.

\section{Acknowledgments}

This work was supported by the National Natural Science Foundation of China (nos. 51575201 and 51405093), Guangxi Key Laboratory of Manufacturing Systems and Advanced Manufacturing Technology, China (no. 14-045-15-006Z), and Natural Science Foundation of Guangxi, China (no. 2015GXNSFBA139232).

\section{References}

[1] F. J. Fahy and E. Lindqvist, "Wave propagation in damped, stiffened structures characteristic of ship construction," Journal of Sound and Vibration, vol. 45, no. 1, pp. 115-138, 1976.

[2] B. R. Mace, "Periodically stiffened fluid-loaded plates, I: response to convected harmonic pressure and free wave propagation," Journal of Sound and Vibration, vol. 73, no. 4, pp. 473-486, 1980.

[3] B. R. Mace, "Periodically stiffened fluid-loaded plates, II: response to line and point forces," Journal of Sound and Vibration, vol. 73, no. 4, pp. 487-504, 1980.

[4] B. R. Mace, "The vibration of plates on two-dimensionally periodic point supports," Journal of Sound and Vibration, vol. 192, no. 3, pp. 629-643, 1996.

[5] D. J. Mead, "Wave propagation in continuous periodic structures: research contributions from Southampton, 1964-1995," Journal of Sound and Vibration, vol. 190, no. 3, pp. 495-524, 1996.

[6] J.-H. Lee and J. Kim, "Analysis of sound transmission through periodically stiffened panels by space-harmonic expansion method," Journal of Sound and Vibration, vol. 251, no. 2, pp. 349366, 2002.

[7] J. Wang, T. J. Lu, J. Woodhouse, R. S. Langley, and J. Evans, "Sound transmission through lightweight double-leaf partitions: theoretical modelling," Journal of Sound and Vibration, vol. 286, no. 4-5, pp. 817-847, 2005. 
[8] J. Legault and N. Atalla, "Numerical and experimental investigation of the effect of structural links on the sound transmission of a lightweight double panel structure," Journal of Sound and Vibration, vol. 324, no. 3-5, pp. 712-732, 2009.

[9] L. Dozio and M. Ricciardi, "Free vibration analysis of ribbed plates by a combined analytical-numerical method," Journal of Sound and Vibration, vol. 319, no. 1-2, pp. 681-697, 2009.

[10] F. X. Xin and T. J. Lu, "Analytical modeling of fluid loaded orthogonally rib-stiffened sandwich structures: sound transmission," Journal of the Mechanics and Physics of Solids, vol. 58, no. 9, pp. 1374-1396, 2010.

[11] F. X. Xin and T. J. Lu, "Analytical modeling of wave propagation in orthogonally rib-stiffened sandwich structures: sound radiation," Computers and Structures, vol. 89, no. 5-6, pp. 507-516, 2011.

[12] V. Hongisto, "Airborne sound insulation of wall structuresmeasurement and prediction methods," Tech. Rep. 56, Laboratory of Acoustics and Audio Signal Processing, Helsinki University of Technology, Espoo, Finland, 2000.

[13] Y. Hamada, Y. Hidaka, and H. Tachibana, "Estimation of random incidence sound transmission loss of multiple structure walls by four-terminal network theory," Journal of Structural and Construction Engineering (Transactions of Architectural Institute of Japan), vol. 362, pp. 1-7, 1986 (Japanese).

[14] B. H. Sharp, "Prediction methods for the sound transmission of building elements," Noise Control Engineering, vol. 11, no. 2, pp. 53-63, 1978.

[15] Q. Gu and J. Wang, "Effect of resilient connection on sound transmission loss of metal stud double panel partitions," Chinese Journal of Acoustics, vol. 2, pp. 113-126, 1983.

[16] F. Fahy and P. Gardonio, Sound and Structural Vibration, Elsevier, Amsterdam, The Netherlands, 2nd edition, 2007.

[17] J. L. Davy, "The sound transmission of cavity walls due to studs," in Proceedings of the INTER-NOISE and NOISE-CON Congress and Conference (InterNoise '93), vol. 4, pp. 975-978, Leuven, Belgium, 1993.

[18] M. L. Rumerman, "Vibration and wave propagation in ribbed plates," Journal of the Acoustical Society of America, vol. 57, no. 2, pp. 370-373, 1975.

[19] G. F. Lin and J. A. M. Garrelick, "Sound transmission through periodically framed parallel plates," Journal of the Acoustical Society of America, vol. 61, no. 4, pp. 1014-1018, 1977.

[20] D. Takahashi, "Sound radiation from periodically connected double-plate structures," Journal of Sound and Vibration, vol. 90, no. 4, pp. 541-557, 1983.

[21] L. Maxit, "Wavenumber space and physical space responses of a periodically ribbed plate to a point drive: a discrete approach," Applied Acoustics, vol. 70, no. 4, pp. 563-578, 2009.

[22] D. J. Mead, "Free wave propagation in periodically supported, infinite beams," Journal of Sound and Vibration, vol. 11, no. 2, pp. 181-197, 1970.

[23] D. J. Mead and K. K. Pujara, "Space-harmonic analysis of periodically supported beams: response to convected random loading," Journal of Sound and Vibration, vol. 14, no. 4, pp. 525-541, 1971.

[24] R. E. Halliwell, T. R. T. Nightingale, A. C. C. Warnock, and J. A. Birta, "Gypsum board walls: transmission loss data," Internal Report IRC-IR-761, Institute for Research in Construction, National Research Council of Canada, Ottawa, Canada, 1998.

[25] J. L. Davy, "Predicting the sound insulation of walls," Building Acoustics, vol. 16, no. 1, pp. 1-20, 2009.
[26] J. L. Davy, "Sound transmission of cavity walls due to structure borne transmission via point and line connections," Journal of the Acoustical Society of America, vol. 132, no. 2, pp. 814-821, 2012.

[27] V. Hongisto, "Sound insulation of doors-Part 1: prediction models for structural and leak transmission," Journal of Sound and Vibration, vol. 230, no. 1, pp. 133-148, 2000.

[28] V. Hongisto, J. Keränen, and M. Lindgren, "Sound insulation of doors-Part 2: comparison between measurement results and predictions," Journal of Sound and Vibration, vol. 230, no. 1, pp. 149-170, 2000.

[29] V. Hongisto, M. Lindgren, and J. Keränen, "Enhancing maximum measurable sound reduction index using sound intensity method and strong receiving room absorption," Journal of the Acoustical Society of America, vol. 109, no. 1, pp. 254-265, 2001.

[30] V. Hongisto, "Case study of flanking transmission through double structures," Applied Acoustics, vol. 62, no. 5, pp. 589-599, 2001.

[31] N. W. Hagood and A. von Flotow, "Damping of structural vibrations with piezoelectric materials and passive electrical networks," Journal of Sound and Vibration, vol. 146, no. 2, pp. 243-268, 1991.

[32] J. J. Hollkamp, "Multimodal passive vibration suppression with piezoelectric materials and resonant shunts," Journal of Intelligent Material Systems and Structures, vol. 5, no. 1, pp. 49-57, 1994.

[33] O. Thorp, M. Ruzzene, and A. Baz, "Attenuation and localization of wave propagation in rods with periodic shunted piezoelectric patches," Smart Materials and Structures, vol. 10, no. 5, pp. 979989, 2001.

[34] O. Thorp, M. Ruzzene, and A. Baz, "Attenuation of wave propagation in fluid-loaded shells with periodic shunted piezoelectric rings," Smart Materials and Structures, vol. 14, no. 4, pp. 594604, 2005.

[35] S. Chen, G. Wang, J. Wen, and X. Wen, "Wave propagation and attenuation in plates with periodic arrays of shunted piezopatches," Journal of Sound and Vibration, vol. 332, no. 6, pp. 1520-1532, 2013.

[36] Y. Xiao, J. Wen, and X. Wen, "Sound transmission loss of metamaterial-based thin plates with multiple subwavelength arrays of attached resonators," Journal of Sound and Vibration, vol. 331, no. 25, pp. 5408-5423, 2012.

[37] H. Zhang, J. Wen, Y. Xiao, G. Wang, and X. Wen, "Sound transmission loss of metamaterial thin plates with periodic subwavelength arrays of shunted piezoelectric patches," Journal of Sound and Vibration, vol. 343, pp. 104-120, 2015.

[38] B. R. Mace, "Sound radiation from fluid loaded orthogonally stiffened plates," Journal of Sound and Vibration, vol. 79, no. 3, pp. 439-452, 1981.

[39] G. P. Mathur, B. N. Tran, J. S. Bolton, and N.-M. Shiau, "Sound transmission through stiffened double-panel structures lined with elastic porous materials," in Proceedings of the 14th DGLR/ AIAA Aeroacoustics Conference, pp. 102-105, Aachen, Germany, May 1992.

[40] A. Spadoni and M. Ruzzene, "Structural and acoustic behavior of chiral truss-core beams," Journal of Vibration and Acoustics, Transactions of the ASME, vol. 128, no. 5, pp. 616-626, 2006.

[41] F. X. Xin, T. J. Lu, and C. Q. Chen, "External mean flow influence on noise transmission through double-leaf aeroelastic plates," AIAA Journal, vol. 47, no. 8, pp. 1939-1951, 2009. 
[42] E. E. Ungar and J. A. Zapfe, Structural Damping, Noise and Vibration Control Engineering: Principles and Applications, John Wiley \& Sons, 2nd edition, 2007.

[43] E. G. Williams, Fourier Acoustics: Sound Radiation and Nearfield Acoustical Holography, Academic Press, 1999. 


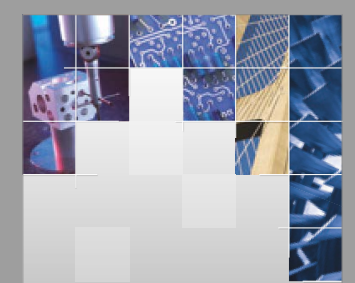

\section{Enfincering}
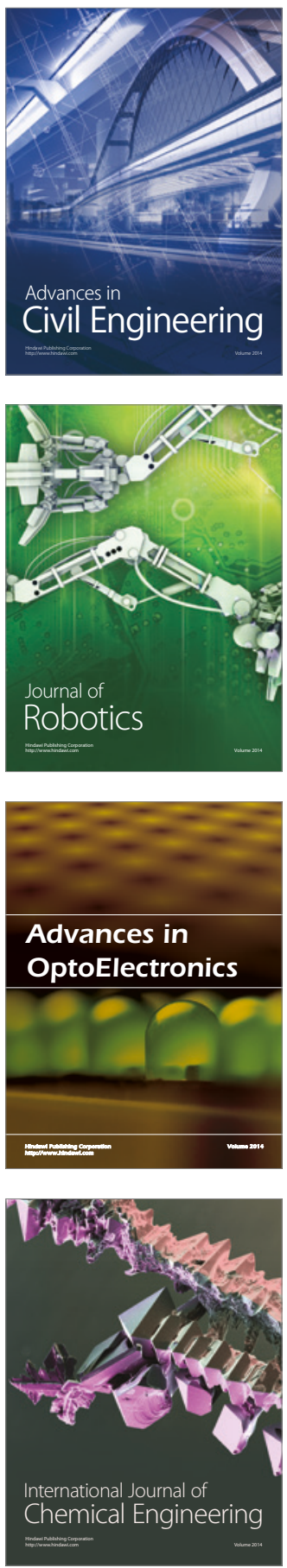

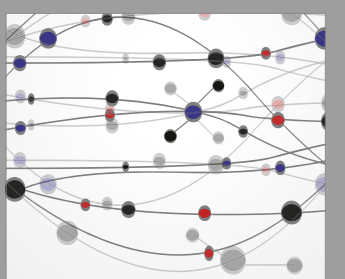

The Scientific World Journal

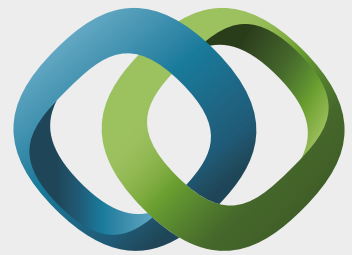

\section{Hindawi}

Submit your manuscripts at

https://www.hindawi.com
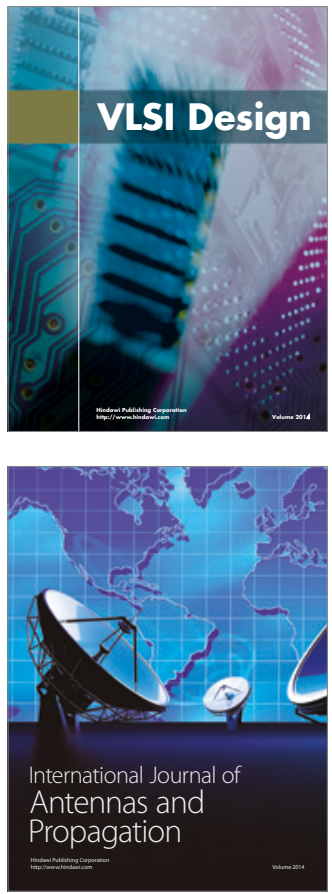

\section{Rotating}

Machinery
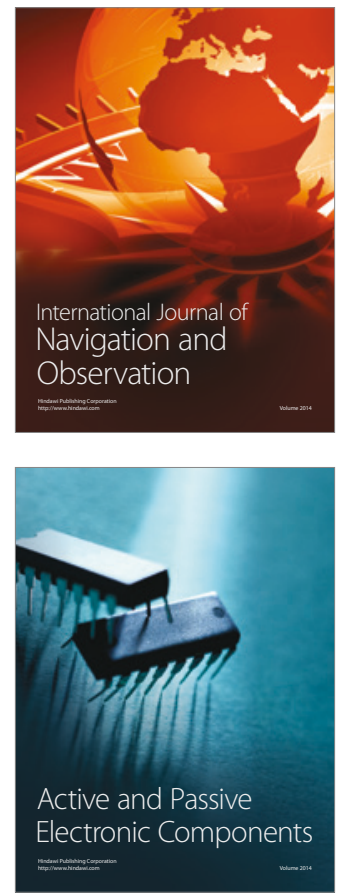
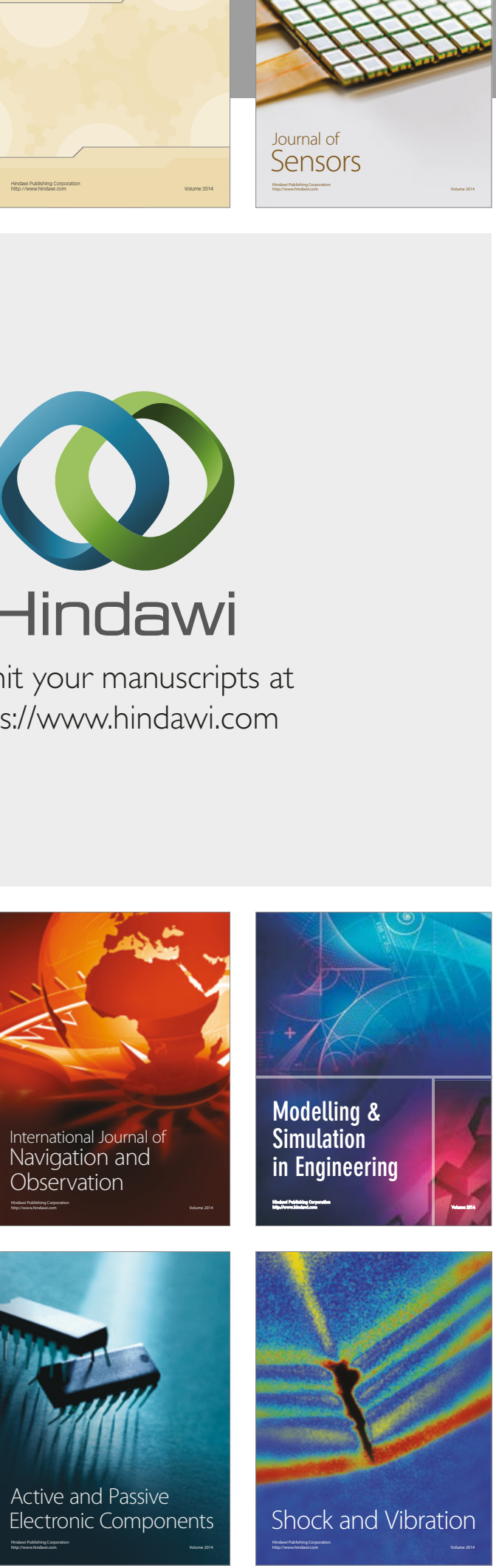
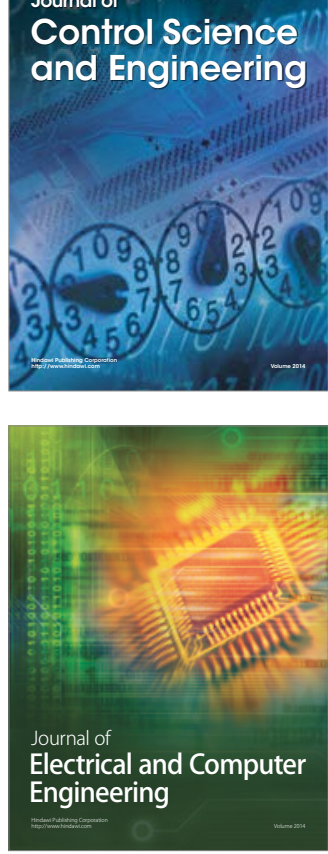

Distributed

Journal of

Control Science

and Engineering
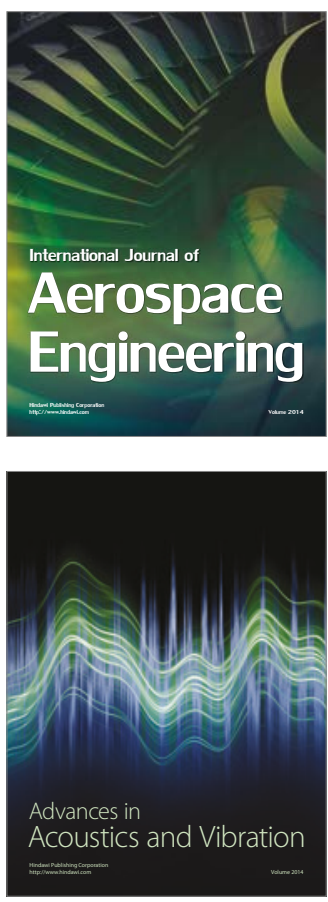

Sensor Networks 